\title{
Research Paper: \\ Meta-analysis Study of Work-related Musculoskeletal Disorders in Iran
}

\author{
Ayoub Parno $^{1}{ }^{\oplus}$, Kourosh Sayehmiri² ${ }^{\circledR}$, Reza Nabi Amjad ${ }^{3} \odot$, Reyhaneh Ivanbagha ${ }^{4}$, Mir Mohammad Hosseini Ahagh ${ }^{5}$, Shiva \\ Hosseini Foladi ${ }^{6},{ }^{*}$ Alireza Khammar ${ }^{7}{ }^{\circ},{ }^{*}$ Mohsen Poursadeqiyan ${ }^{8,9}$ [C]
}

\author{
1. Student Research Committee, Kermanshah University of Medical Sciences, Kermanshah, Iran. \\ 2. Department of Biostatistic, Faculty of Health, Ilam University of Medical Scienses, Ilam, Iran. \\ 3. Non-communicable Diseases Research Center, Alborz University of Medical Sciences, Karaj, Iran. \\ 4. Department of Midwife, School of Nursing, Khalkhal University Medical Sciences, Khalkhal, Iran. \\ 5. Department of Public Health, Khalkhal University of Medical Sciences, Khalkhal, Iran. \\ 6. Department of Health Services Management, Student Research committee, Isfahan University of Medical Sciences, Isfahan, Iran. \\ 7. Department of Occupational Health, Zabol Medicinal Plants Research Center, Zabol University of Medical Sciences, Zabol, Iran. \\ 8. Department of Occupational Health Engineering, School of Health, Torbat Heydariyeh University of Medical Sciences, Torbat Heydariyeh, Iran \\ 9. Health Sciences Research Center, Torbat Heydariyeh University of Medical Sciences, Torbat Heydariyeh, Iran.
}

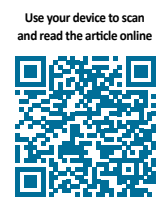

Crtation Parno A, Sayehmiri K, Nabi Amjad R, Ivanbagha R, Hosseini Ahagh MM, Hosseini Foladi S, et al. [Meta-Analysis Study of Work-Related Musculoskeletal Disorders in Iran (Persian)]. Archives of Rehabilitation. 2020; 21(2):182-205. https://doi. org/10.32598/RJ.21.2.2444.4

doli https://doi.org/10.32598/RJ.21.2.2444.4

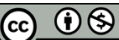

Received: 07 Dec 2018 Accepted: 14 Sep 2019 Available Online: $01 \mathrm{Jul} 2020$

Keywords:

Ergonomics, Upper extremity, Lower extremity, Metaanalysis, Systematic review

\section{A B STRACT}

Objective Work-related musculoskeletal disorders are one of the major causes of lost working time, increased costs, human injuries and burnout. The International Labor Organization estimates that about 160 million work-related illnesses occur worldwide each year, where the highest prevalent illnesses are musculoskeletal disorders. Several studies have been conducted In Iran to investigate the prevalence of these disorders, each providing different statistics. Therefore, considering the importance of these disorders, it is necessary to obtain accurate results with high statistical power and validate them. The aim of this study was to investigate the prevalence of work-related musculoskeletal disorders in Iran in order to obtain more accurate results by conducting a meta-analysis.

Materials \& Methods For this meta-analysis, searching was conducted in SID, MagIran, IranMedex, Google Scholar, Scopus, Web of Science, and PubMed databases using the keyword "work-related musculoskeletal disorders" in both Persian and English on studies conducted during 2001-2016 in various cities of Iran. Inclusion criteria were: investigating the prevalence of musculoskeletal disorders in a period of one year, being a cross-sectional or descriptive-analytical study, publication in Persian or English, availability of full-texts, assessment using standard methods (e.g. Nordic Musculoskeletal Questionnaire) and considering the prevalence of musculoskeletal disorders throughout the body (upper and lower extremities). The heterogeneity of the articles was evaluated using the 12 statistics and the data were analyzed in STATA software using meta-analysis and random-effects model.

Results In this study, 45 papers on work-related musculoskeletal disorders were studied. The overall sample size of these studies was 9813, with a mean number of 218 for each study. Most of studies were descriptive-analytical and cross-sectional. In all of them, the prevalence of musculoskeletal disorders in different parts of the body had been investigated. According to their results, the most prevalent workrelated musculoskeletal disorder in lower extremity was low back pain $(0.49 \%)$ and in upper extremity, it was neck pain (39.3\%). The prevalence of musculoskeletal disorders in other parts of the body were $39.32 \%$ in the neck, $36.9 \%$ in shoulders, $36.8 \%$ in the back, $34 \%$ in the wrist, $26.9 \%$ in legs, $20.5 \%$ in thighs and hips, and $16.2 \%$ in elbows.

\section{* Corresponding Authors:}

\section{Mohsen Poursadeqiyan}

Address: Department of Occupational Health Engineering, School of Health, Torbat Heydariyeh University of Medical Sciences, Torbat Heydariyeh, Iran.

Tel: +98 (51)52226011

E-Mail: mo.poursadeghiyan@uswr.ac.ir

\section{Alireza Khammar}

Address: Zabol Medicinal Plants Research Center, Department of Occupational Health, School of Health, Zabol University of Medical Sciences, Zabol, Iran

Tel: +98 (54) 32232190

E-Mail: alireza.khammar@zbmu.ac.ir 


\begin{abstract}
Conclusion There is a relatively high prevalence of musculoskeletal disorders in lower extremity (lower back) and upper extremity (neck and shoulders) of workers in Iran which is more acute compared to other countries, and need further studies to determine its causes. Therefore, more attention should be paid to the of the health of employees, and ergonomic intervention programs should be designed by managers and officials of organizations to prevent musculoskeletal disorders at workplaces. Since the workers in Iran are young, there may not be high musculoskeletal complaints at the present time, but in the future it will create some problems for them. Therefore, reducing working hours, changing workplace design such that the design of ordered chairs tables be in accordance with ergonomics principles and body dimensions, scheduling time for employees to exercise, and providing training courses for them to maintain proper physical condition at work are suggested as strategies to reduce the risk of musculoskeletal disorders.
\end{abstract}

\section{English Version}

\section{Introduction}

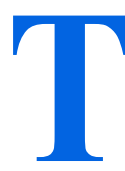

he National Institute for Occupational Safety and Health (NIOSH) defines musculoskeletal disorders as a group of conditions that affect nerves, tendons, muscles, and supporting structures such as intervertebral discs $[1,2]$.

These disorders are the main causes of lost working time, increased costs, human injuries, burnout and the imposition of direct and indirect costs on the health system [2-7]. In Japan, the growth of musculoskeletal disorders from the 1960s to the 1980s was often seen among people who worked as typewriters, telephone operators, and assembly line workers, and most of them had discomfort in the hands, arms, and shoulders. In the Scandinavian countries, these problems became apparent in the 1980s and were often associated with back pain among white-collar workers and neck, wrist and shoulder injuries among blue-collar workers. Nordic studies of factors causing neck and arm pains increased the accuracy of the study [8]. According to studies in Europe, there are about 40 million workers with musculoskeletal disorders (more than $30 \%$ of workers) whose cost is equal to $0.5-2 \%$ of EU gross domestic product [9].

Many studies have been done in this regard in Iran. The results of the study of musculoskeletal disorders and factors associated with the development of these disorders in 94 workers in the painting industry showed that the symptoms of musculoskeletal disorders in some areas of the body such as back, wrist, knee and shoulder are more than in other parts of the body [10]. The results of ergonomic risk assessment study and the prevalence of musculoskeletal disorders among 99 people working in the auto repair industry showed that the most symptoms of musculoskeletal disorders was in the lower back area (62.6\%); most complaints was related to the wrist area (64.6\%), and (55.5\%) at high risk for pain and discomfort [11].
Choobineh et al. [12] in a study in Isfahan on 1439 weavers showed that the prevalence of these disorders was high among carpet weavers and was mainly seen in back $(45.2 \%)$, hips and thighs $(1.6 \%)$, knees $(34.6 \%)$ and ankles $(23.7 \%)(34.6 \%)$. In another study conducted by Mostaghasi et al. [13] on 92 workers in the food industry in Yazd, they reported a low back pain prevalence of $44.6 \%$ [13]. Due to the need to know the latest statistics of musculoskeletal disorders for health planning and considering that many studies in this field need accreditation [14], this metaanalysis study aimed to determine the prevalence of workrelated musculoskeletal disorders in Iran based on location.

\section{Materials \& Methods}

This is a meta-analysis study. A search was conducted in SID (Jihad University Database), MagIran, MedexIran, Google Scholar, Scopus, WOS and Pubmed databases among studies conducted between 2001 and 2016 in different cities of Iran, by using the keyword "prevalence of musculoskeletal disorder" in both Persian and English. Criteria for inclusion of studies in the study were: investigating the prevalence of musculoskeletal disorders in a period of one year, being a cross-sectional or descriptive-analytical study, publication in Persian or English, availability of full-texts, assessment using standard methods (e.g. Nordic Musculoskeletal Questionnaire) and considering the prevalence of musculoskeletal disorders throughout the body (upper and lower extremities). Figure 1 presents a flowchart for the process of selecting articles for meta-analysis.

Considering that the prevalence of musculoskeletal disorders and the sample size were present in all articles, a binomial distribution was used to calculate the variance and the weighted mean was used to combine the prevalence in the studies. Each study was weighted according to its inverse variance. On the other hand, since there was a large difference in the prevalence rates between the studies and the heterogeneity was more than $97 \%$, it was considered as a severe heterogeneity according to the following classifi- 


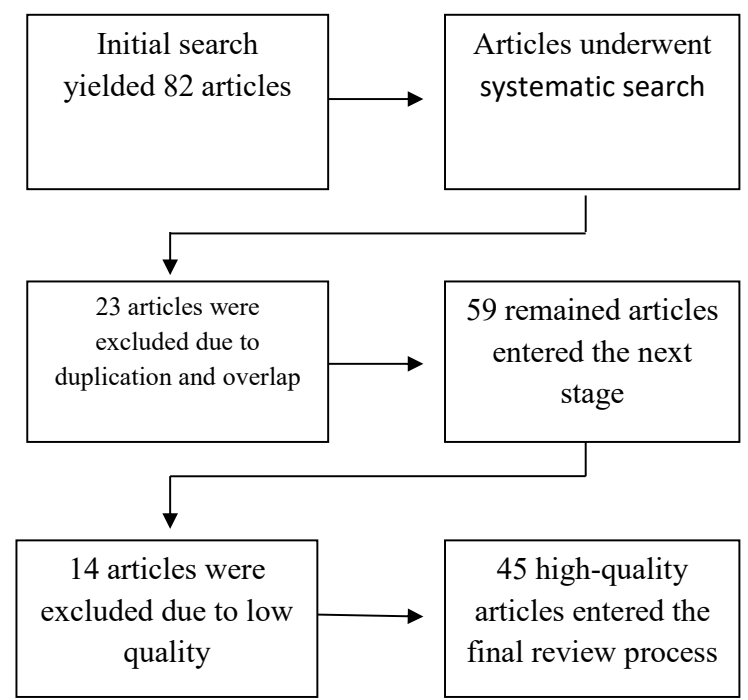

Figure 1. The process of selecting studies for meta-analysis

Rehabilitation

cation method: $\mathrm{I} 2<25 \%=$ partial heterogeneity, $\mathrm{I} 2$ between 25 and $75 \%=$ moderate heterogeneity, and $\mathrm{I} 2>75 \%=$ severe heterogeneity. Random-effects model was used for assessment. According to this model, it is assumed that the differences are due to using different sampling method and variation in measured parameters [42].
Moreover, according to the type of study data, the plotting of graphs and determining the publication bias for all disorders were not performed. Meta-Regression was used to calculate the relationship between the prevalence of musculoskeletal disorders and the year of study and sample size. Data analysis was performed in STATA V.10 software.

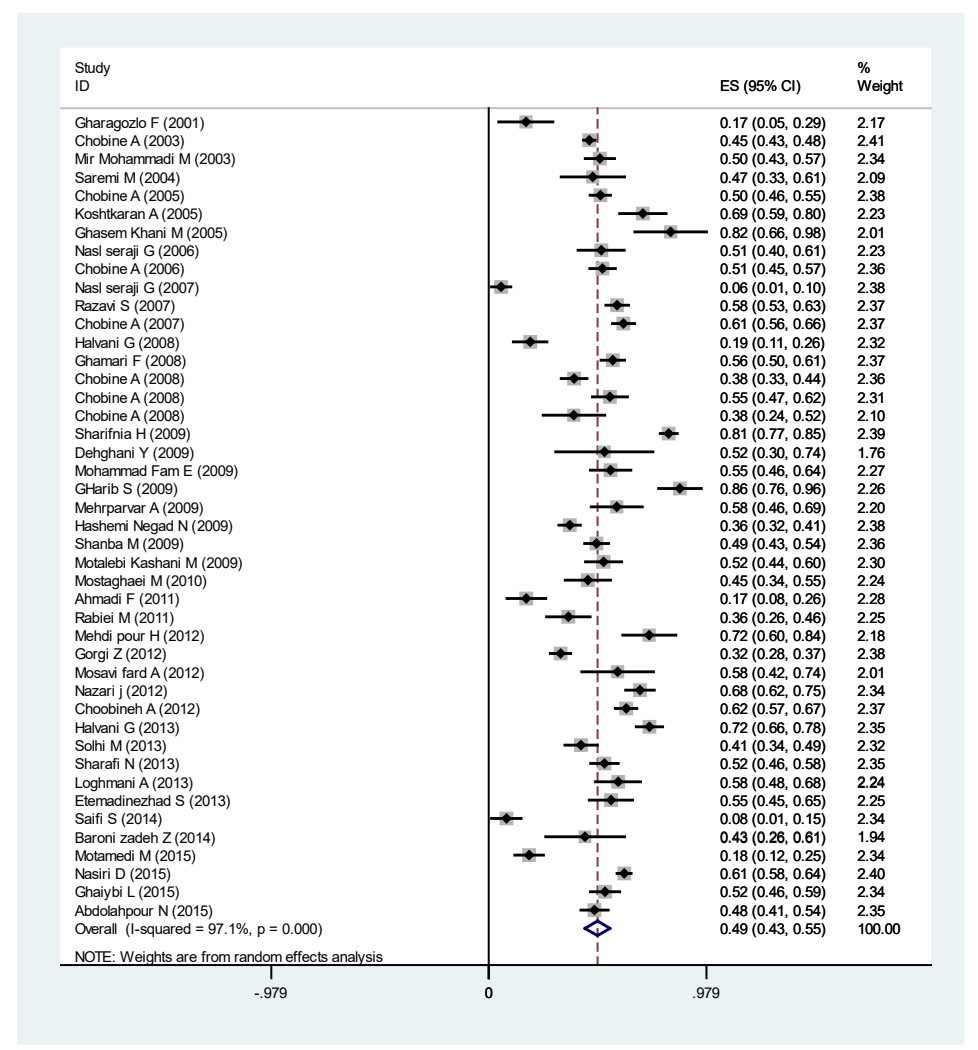

Archives of

Figure 2. Studies reported the prevalence of lower back pain in lower extremity presented based on a random-effects model 
Table 1. Prevalence of musculoskeletal disorders in Iran

\begin{tabular}{|c|c|c|c|c|c|c|c|}
\hline \multirow{2}{*}{ Location } & \multirow{2}{*}{ Year } & \multicolumn{2}{|c|}{ Sample Size } & \multicolumn{4}{|c|}{ Prevalence of Musculoskeletal Disorders in Upper Extremity } \\
\hline & & Table 1 & Table 2 & Neck & Shoulder & Wrist & Elbow \\
\hline Tehran & 2003 & 220 & 220 & 0.24 & 0.17 & 0.23 & - \\
\hline Tehran & 2004 & 47 & 47 & 0.83 & 0.62 & 0.468 & 085 \\
\hline Tehran & 2009 & 115 & 115 & 0.2608 & 0.4434 & 0.4 & 0.148 \\
\hline Kermanshah & 2001 & 36 & 36 & 0.167 & - & 0.445 & 0.167 \\
\hline Tehran & 2009 & 20 & 20 & 0.22 & 0.32 & 0.32 & 0.154 \\
\hline Yazd & 2008 & 102 & 102 & 088 & 029 & 009 & - \\
\hline Yazd & 2009 & 72 & 72 & 0.465 & 0.203 & 0.124 & 051 \\
\hline Yazd & 2010 & 92 & 92 & 099 & 0.174 & 0.228 & \\
\hline Shiraz & 2006 & 288 & 288 & 0.582 & 0.596 & 0.488 & 0.289 \\
\hline Tehran & 2005 & 22 & 22 & 0.364 & 0.727 & 0.727 & 0.5 \\
\hline Urmia & 2006 & 89 & 89 & 0.258 & 0.404 & 0.438 & 045 \\
\hline Tekab & 2007 & 110 & 110 & - & - & - & - \\
\hline Shiraz & 2007 & 375 & 375 & 0.519 & 0.517 & 0.471 & 0.229 \\
\hline Tehran & 2009 & 145 & 145 & 0.178 & 0.483 & 0.331 & 076 \\
\hline Isfahan & 2003 & 1439 & 1439 & 0.352 & 0.478 & 0.382 & 0.192 \\
\hline Qom & 2008 & 47 & 47 & 0.149 & 0.17 & 0.298 & 0.128 \\
\hline Qom & 2008 & 268 & 268 & 0.183 & 0.224 & 0.284 & 056 \\
\hline Tehran & 2009 & 332 & 332 & 0.38 & 0.354 & 0.305 & 0.135 \\
\hline Arak & 2008 & 334 & 334 & - & - & - & - \\
\hline Shiraz & 2005 & 454 & 454 & 0.2 & 0.32 & 0.35 & - \\
\hline Amol & 2009 & 400 & 400 & 0.5 & 0.35 & 0.295 & - \\
\hline Sabzevar & 2007 & 364 & 364 & 0.319 & 0.343 & 0.355 & - \\
\hline Kerman & 2009 & 384 & 384 & 046 & 0.169 & 0.195 & - \\
\hline Meybod & 2009 & 54 & 54 & - & 0.389 & 0.426 & 0.148 \\
\hline Isfahan & 2009 & 50 & 50 & 0.24 & 0.44 & 0.28 & 0.3 \\
\hline Shiraz & 2008 & 156 & 156 & 0.231 & 0.263 & 0.237 & 09 \\
\hline Shiraz & 2005 & 75 & 75 & 0.773 & 0.747 & 0.677 & 0.627 \\
\hline Qazvin & 2012 & 54 & 54 & 0.537 & 0.537 & 0.593 & 0.371 \\
\hline Yazd & 2013 & 220 & 220 & 0.814 & 0.295 & 0.209 & 0.140 \\
\hline Rafsanjan & 2012 & 400 & 400 & 0.323 & 0.262 & 0.190 & 070 \\
\hline Hamedan & 2011 & 71 & 71 & 0.497 & 0.296 & 0.478 & 014 \\
\hline Babol & 2014 & 64 & 64 & 0.254 & - & - & - \\
\hline Hamedan & 2014 & 30 & 30 & 0.633 & 0.133 & 0.100 & 067 \\
\hline Isfahan & 2015 & 138 & 138 & 0.261 & - & - & - \\
\hline Tehran & 2013 & 176 & 176 & 0.597 & 0.420 & 0.324 & 0.165 \\
\hline Sari & 2015 & 940 & 940 & 0.505 & 0.294 & 0.330 & 0.146 \\
\hline Tekab & 2015 & 222 & 222 & 0.514 & 0.432 & 0.158 & 072 \\
\hline Iran & 2015 & 254 & 254 & 0.346 & 0.287 & 0.236 & 0.173 \\
\hline Sanandaj & 2013 & 250 & 250 & 0.388 & 0.496 & 0.396 & 0.200 \\
\hline Karaj & 2012 & 36 & 36 & 0.530 & 0.280 & 0.390 & - \\
\hline Guilan & 2011 & 92 & 92 & 0.430 & 0.250 & 0.250 & - \\
\hline Isfahan & 2013 & 91 & 91 & 0.692 & 0.352 & 0.285 & 0.132 \\
\hline
\end{tabular}




\begin{tabular}{|c|c|c|c|c|c|c|c|}
\hline \multirow{2}{*}{ Location } & \multirow{2}{*}{ Year } & \multicolumn{2}{|c|}{ Sample Size } & \multicolumn{4}{|c|}{ Prevalence of Musculoskeletal Disorders in Upper Extremity } \\
\hline & & Table 1 & Table 2 & Neck & Shoulder & Wrist & Elbow \\
\hline Tabriz & 2012 & 200 & 200 & 0.787 & 0.489 & 0.553 & 0.277 \\
\hline Sari & 2013 & 100 & 100 & 0.310 & 0.370 & 0.200 & 0.240 \\
\hline Iran & 2012 & 385 & 385 & 0.486 & 0.455 & 0.481 & 0.158 \\
\hline \multirow{2}{*}{ Location } & \multirow{2}{*}{ Year } & \multicolumn{6}{|c|}{ Prevalence of Musculoskeletal Disorders in Lower Extremity } \\
\hline & & Back & & Hips and thighs & Knee & & Ankle \\
\hline Tehran & 2003 & 09 & & 09 & - & & 0.23 \\
\hline Tehran & 2004 & 0.511 & & 0.255 & 0.234 & & - \\
\hline Tehran & 2009 & 0.452 & & 0.1217 & 0.5043 & & 0.200 \\
\hline Kermanshah & 2001 & 0.501 & & & 056 & & 056 \\
\hline Tehran & 2009 & 0.38 & & 0.17 & 0.47 & & 0.374 \\
\hline Yazd & 2008 & - & & 029 & 0090 & & - \\
\hline Yazd & 2009 & - & & - & - & & - \\
\hline Yazd & 2010 & - & & - & - & & - \\
\hline Shiraz & 2006 & 0.547 & & 0.216 & 0.394 & & 0.317 \\
\hline Tehran & 2005 & 0.273 & & 0.344 & 0.591 & & 0.227 \\
\hline Urmia & 2006 & 0.124 & & 067 & 0.337 & & 011 \\
\hline Tekab & 2007 & - & & 0.555 & 0.436 & & 0.455 \\
\hline Shiraz & 2007 & 0.546 & & 0.307 & 0.581 & & 0.590 \\
\hline Tehran & 2009 & 0.441 & & 097 & 0.193 & & 0.324 \\
\hline Isfahan & 2003 & 0.377 & & 0.16 & 0.346 & & 0.237 \\
\hline Qom & 2008 & - & & 0.128 & 0.298 & & 085 \\
\hline Qom & 2008 & 0.146 & & 0.134 & 0.362 & & 0.213 \\
\hline Tehran & 2009 & 0.383 & & 0.124 & 0.401 & & 0.154 \\
\hline Arak & 2008 & - & & & 0.612 & & 0.539 \\
\hline Shiraz & 2005 & - & & 0.170 & 0.480 & & 0.374 \\
\hline Amol & 2009 & - & & & 0.365 & & - \\
\hline Sabzevar & 2007 & 0.436 & & 0.190 & 0.44 & & 0.226 \\
\hline Kerman & 2009 & - & & - & - & & 0.333 \\
\hline Meybod & 2009 & - & & - & - & & - \\
\hline Isfahan & 2009 & 046 & & 0.48 & 0.66 & & 0.24 \\
\hline Shiraz & 2008 & 0.212 & & - & 0.429 & & 0.109 \\
\hline Shiraz & 2005 & 0.653 & & 0.747 & 0.251 & & 0.160 \\
\hline Qazvin & 2012 & 0.722 & & 0.704 & 0.593 & & 0.371 \\
\hline Yazd & 2013 & - & & - & - & & - \\
\hline Rafsanjan & 2012 & 0.162 & & 0.114 & 0.270 & & 065 \\
\hline Hamedan & 2011 & 0.239 & & - & 0.141 & & - \\
\hline Babol & 2014 & 079 & & - & - & & - \\
\hline Hamedan & 2014 & 0.300 & & - & 0.200 & & 0.100 \\
\hline Isfahan & 2015 & 0.304 & & - & 0.116 & & - \\
\hline
\end{tabular}




\begin{tabular}{cccccc}
\hline & & \multicolumn{3}{c}{ Prevalence of Musculoskeletal Disorders in Lower Extremity } \\
\cline { 3 - 6 } Location & Year & Back & Hips and thighs & Knee & Ankle \\
\cline { 3 - 6 } & & 0.392 & 0.176 & 0.347 & 0.222 \\
\hline Tehran & 2013 & 0.199 & 0.189 & 0.272 & 0.282 \\
\hline Sari & 2015 & 0.613 & 0.153 & 0.230 & 0.140 \\
\hline Tekab & 2015 & 0.259 & 0.157 & 0.468 & 0.232 \\
\hline Iran & 2015 & 0.424 & - & 0.396 & 0.260 \\
\hline Sanandaj & 2013 & - & - & 0.670 & - \\
\hline Karaj & 2012 & - & 0.108 & 0.195 & 0863 \\
\hline Guilan & 2011 & 0.341 & 077 & 0.418 & 0.132 \\
\hline Isfahan & 2013 & 0.532 & 0.170 & 0.489 & 0.638 \\
\hline Tabriz & 2012 & 0.230 & 0.260 & 0.450 & 0.250 \\
\hline Sari & 2013 & 0.540 & 0.291 & 0.458 & 0.597 \\
\hline Iran & 2012 & & & & \\
\hline
\end{tabular}

\section{Results}

In the studies, overall sample size was 9813 with a mean number of 218 samples in each study. The mean age and work experience of samples in these studies were between 7.91-33.54 and 6.79-10.27 years, respectively. Based on the results shown in Table 1, the overall prevalence of musculoskeletal disorders in the upper extremity was estimated to be $39.3 \%$ in the neck, $36.9 \%$ in the shoulder, $16.2 \%$ in the elbow, $34 \%$ in the wrist and $36.8 \%$ in the back. Moreover, the overall prevalence of musculoskeletal disorders in the lower extremity was estimated to be $49 \%$ in the lower back, $20.5 \%$ in hips and thighs, $39.2 \%$ in the knee, and $26.9 \%$ in the ankle.

Regarding the prevalence of disorders based on occupation, the highest back pain prevalence was reported in office workers with a prevalence of $53 \%$ followed by hospital staff (medicine, dentistry, nursing, etc.) with a prevalence of 50\%; the highest prevalence of hip and thighs pain in office workers $(27.9 \%)$; the highest prevalence of knee pain in computer users (47.8\%); the highest prevalence of ankle pain in office workers $(55.8 \%)$; the highest prevalence of shoulder pain in computer users (40.9\%); the highest prevalence of neck pain

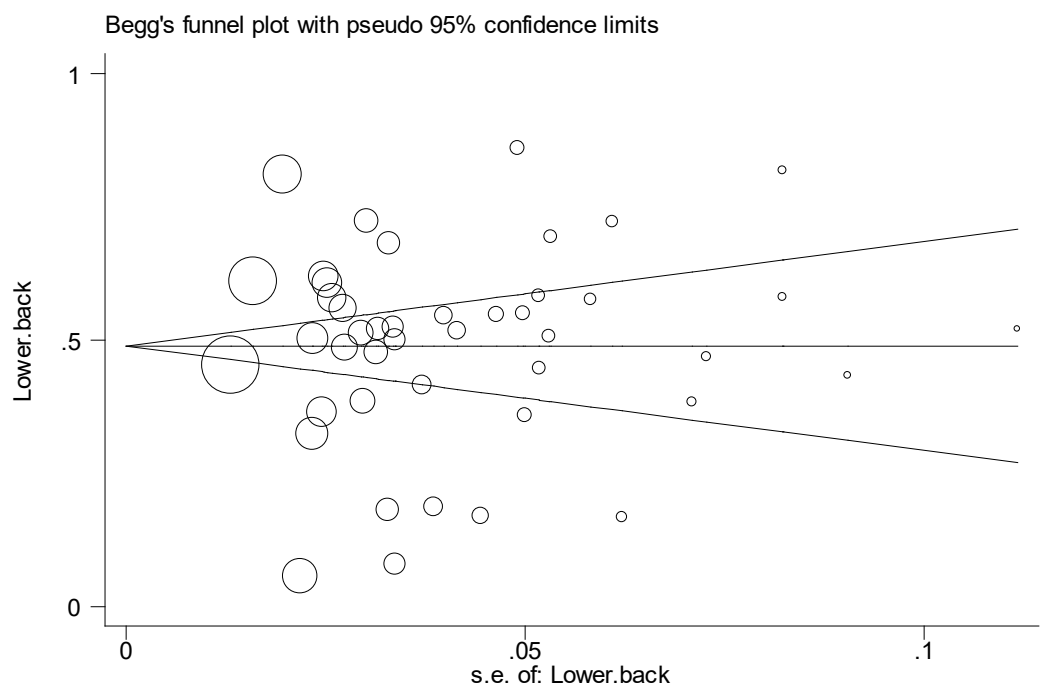

Rehabilitation

Figure 3. Funnel plot of publication bias for the prevalence of lower back pain in Iran during 2001-2016. According to this chart, the prevalence was not significant. 


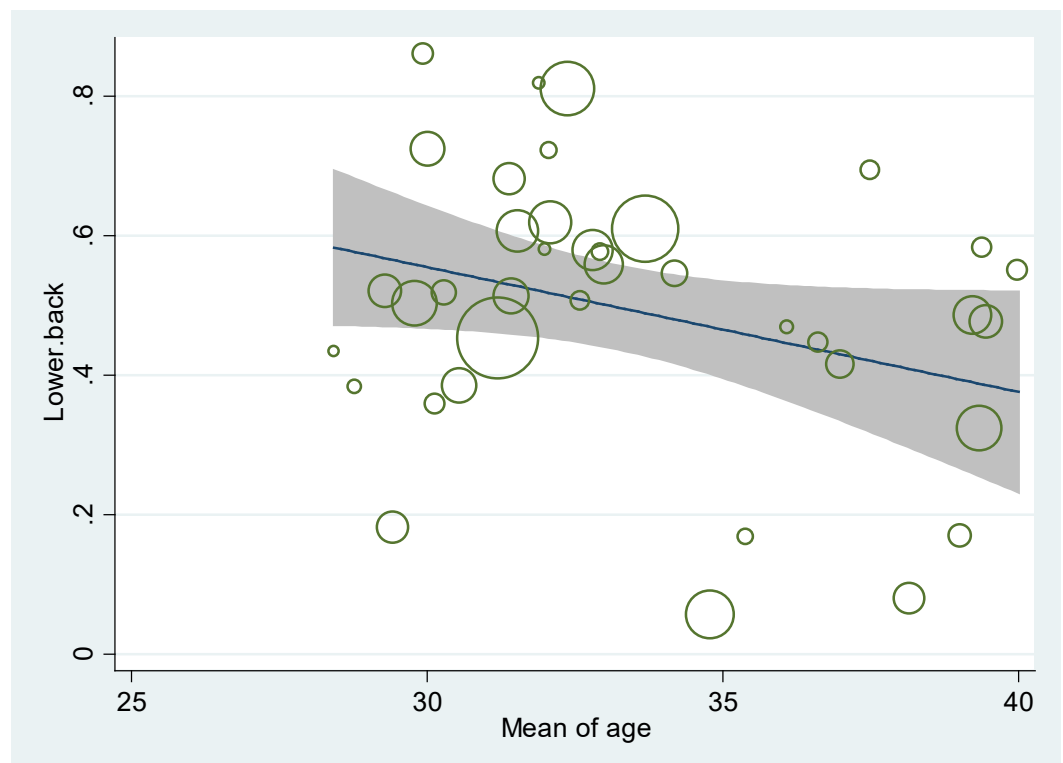

Archives of

Figure 4. Meta-regression scatterplot of the relationship between the prevalence of lower back pain and age. According to this chart, the relationship was not significant.

(49.4\%) and hand pain (37.7\%) in medical staff; the highest prevalence of elbow pain in office workers (22.8\%); and the highest back pain prevalence in computer users $(47.1 \%)$ these results are shown in Figures 2-6.

\section{Discussion}

The results of this study showed a high prevalence of musculoskeletal disorders, especially in the lower back. The highest overall prevalence of work-related musculoskeletal disorders was in the neck (39.3\%) and shoulder (36.9\%). Azizipour et al. reported the prevalence of lower back pain in Iran as 51.6\% [43]. Louw et al. reported a 50\% prevalence of lower back pain in African workers [44], which is consistent with the results of the present study. Hoe et al. (2012) also reported that lower back pain is a major problem in the

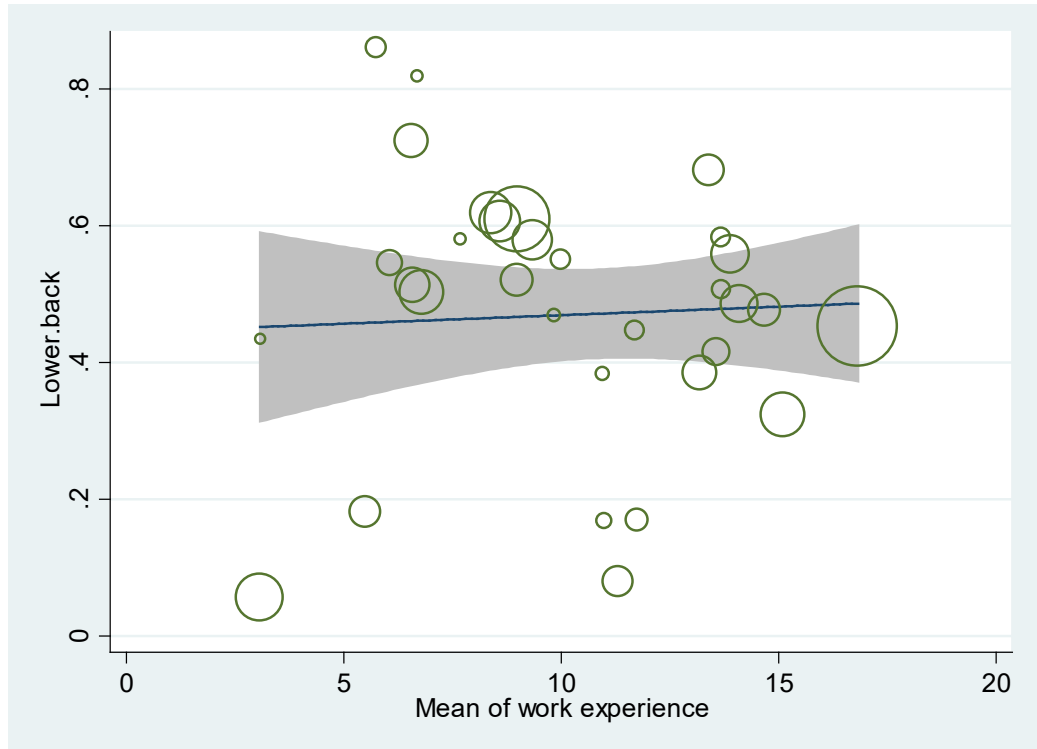

\section{Rehabilitation}

Figure 5. Meta-regression scatterplot of the relationship between the prevalence of lower back pain and work experience. According to this chart, the relationship was not significant. 


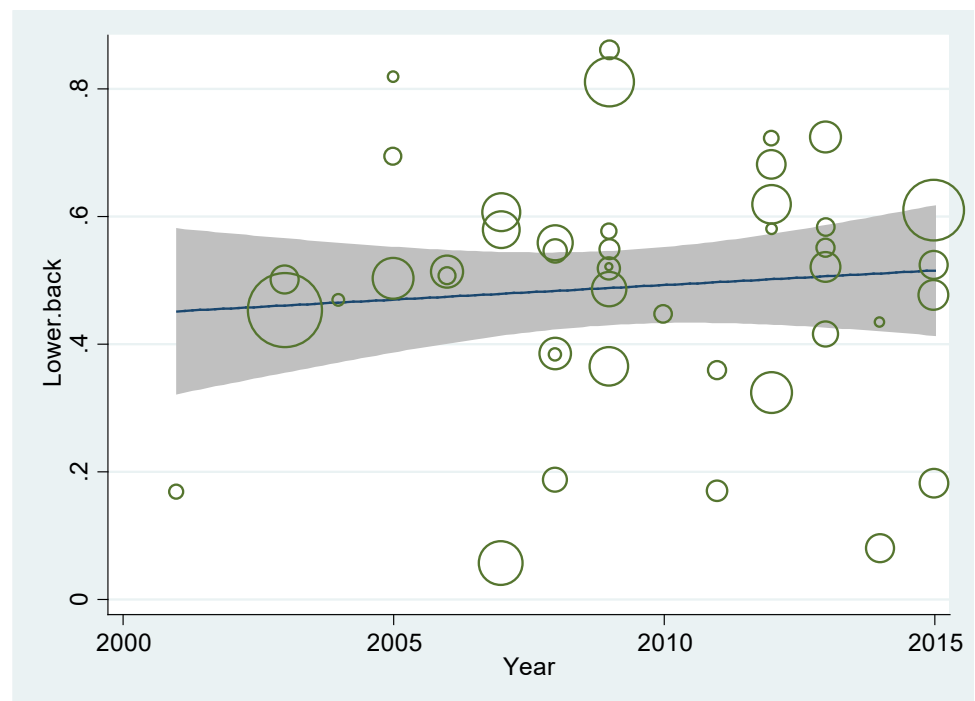

Rehabilitation

Figure 6. Meta-regression scatterplot of the relationship between the prevalence of lower back pain and academic year. Larger circles indicate larger sample sizes.

world. According to them, the prevalence of one-year global musculoskeletal problems was between 22-65\% [45].

In our study, although the maximum rate was $57.5 \%$, it was almost similar to the results of other countries in Hoe et al.'s study; the minimum prevalence reported in our study was almost twice that of their study. Therefore, it can be acknowledged that this problem has been more acute among Iranian employees and needs further studies to investigate the causes of this prevalence. Mehrparvar et al. showed that the prevalence of musculoskeletal disorders in the lower back, shoulder, neck and wrist areas were $44.6 \%, 17.4,9.9$, and $22.2 \%$, respectively, which indicates a high prevalence of these disorders in the lower back [13].

This is also consistent with the results of our study. Osborne et al. (2012) reported that the prevalence of musculoskeletal disorders of any kind during the life of farmers was $90.6 \%$; the prevalence of these disorders in the upper extremity was in the range of 3.6-71.4\% and in the lower extremity as $10.4-41 \%$ [46]. However, in this study, low back pain with a prevalence of $49 \%$ was the highest prevalent musculoskeletal disorder which was more prevalent in office workers (53\%). Therefore, more attention should be paid to the health of employees, and ergonomic intervention programs should be designed by managers and officials of organizations to prevent musculoskeletal disorders related at workplaces.

The prevalence of musculoskeletal disorders in the upper extremity was higher in Tehran and Shiraz cities and lower in Yazd city, which is consistent with the results of Azizpour et al. [43]. Moreover, the prevalence of musculoskeletal disorders in the upper extremities was higher in studies conducted in 2005 than in other years. The highest prevalence of musculoskeletal disorders in Tehran was observed in a study conducted on the employees of one of the household appliance manufacturing industries. The prevalence of musculoskeletal disorders reported in one year in the upper extremity in the study by Mir Mohammadi et al. (2004) was $24 \%$ in the neck, $17 \%$ in the shoulder and arms, $9 \%$ in the upper back, and $50 \%$ in the lower back [17] which is consistent with the results of our study. On the other hand, the prevalence of low back pain was not significantly related to the age factor.

Overall, it can be said that ergonomic design of workplaces and ergonomic training reduces the risk of work-related musculoskeletal disorders, especially in the upper extremity including lower back. Therefore, ergonomic and occupational health training based on the prevention of such disorders [47] is recommended. It is suggested that in future researches on the prevalence of musculoskeletal diseases, a valid shortened form of questionnaire in Persian be used $[48,49]$ and more detailed studies should be performed to evaluate pain and discomfort [50].

\section{Conclusion}

In this study, the highest injury in all work groups was in the lower extremity, especially in the lower back (49\%) and knee (39.2\%); in the upper extremity, musculoskeletal disorders were higher in the neck. Considering the age, work 
experience, academic year, and physical ability of individuals to employ them in different departments, ergonomic training of employees in the field of the best correct way to perform work and the best physical condition while working or sitting, is one of strategies that can play an effective role in preventing musculoskeletal disorders.

Accordingly, it is suggested that ergonomic and occupational health intervention programs be implemented to control or eliminate the risk factors for occupational diseases in various jobs. Ergonomic design of workplaces and ergonomic intervention programs can reduce the risk of work-related musculoskeletal disorders in the upper and lower extremities. Since the employees/workers in Iran are young, there may not be high musculoskeletal complaints at the present time, but in the future it will create some problems for them.

Therefore, reducing working hours and changing workplace design such that the design of ordered chairs and tables be in accordance with ergonomics principles and body dimensions. The use of appropriate footrests, scheduling time for employees to exercise, and providing training courses for them to maintain proper physical condition at work are also suggested as strategies to reduce the risk of musculoskeletal disorders. Most of the studies were crosssectional and used self-report tools to assess the prevalence of musculoskeletal disorders.

Lack of access to the full text of all articles, lack of a specific framework for reporting published articles, lack of access to theses, and low quality and quantity of databases were other limitations of the present study. This study, like other studies had some limitations and obstacles, such as: lack of access to the full text of all articles, lack of a specific framework for reporting published articles, lack of access to dissertation results and low quality and quantity of databases.

\section{Ethical Considerations}

Compliance with ethical guidelines

All ethical principles are considered in this article.

Funding

This research did not receive any grant from funding agencies in the public, commercial, or non-profit sectors.

Authors' contributions

All authors contributed in preparing this article.

\section{Conflicts of interest}

The authors declare no conflict of interest 


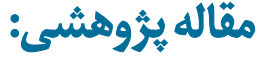 فراتحليل شيوع اختلالات اسكلتى ـعضلانى ناشى از كار در ايران
}

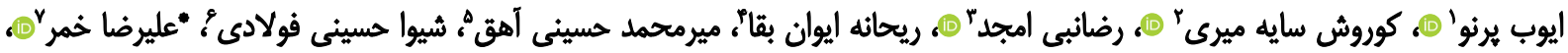

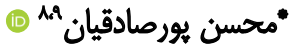

1. كميته تحقيقات دانشجويى، دانشكاه علوم يزشكى كرمانشاه، كرمانشاه، ايران

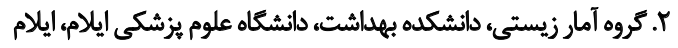

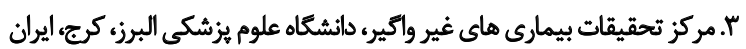

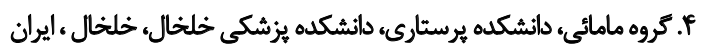

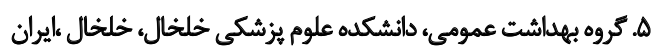

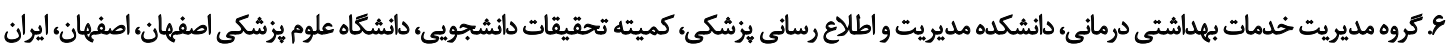

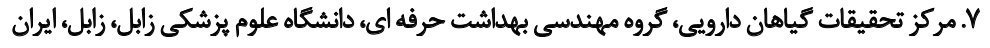

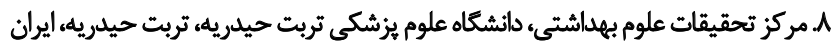

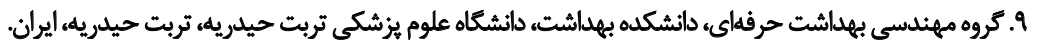

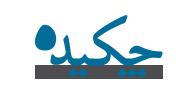

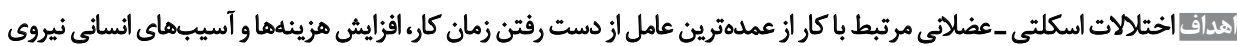

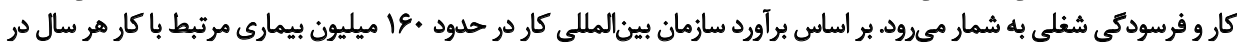

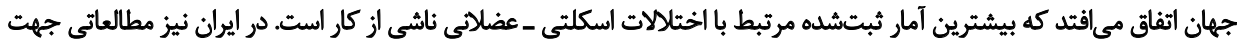

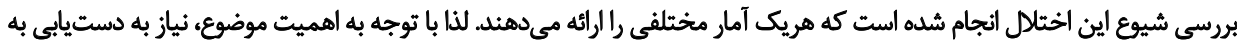

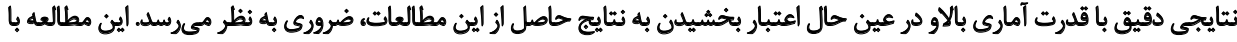

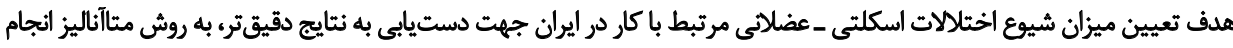

شده است.

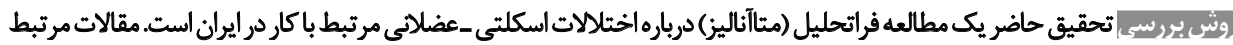

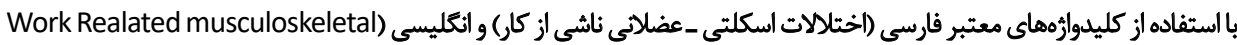

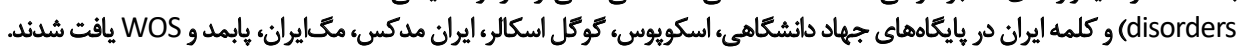

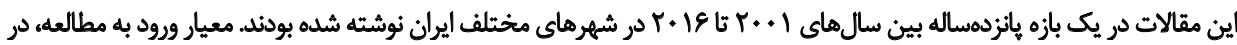

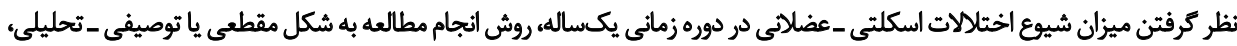

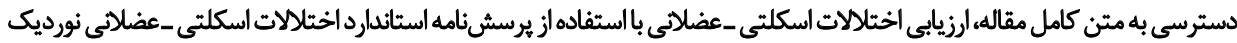

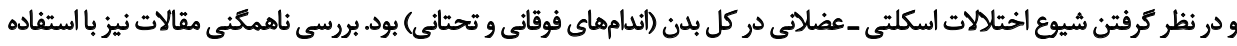

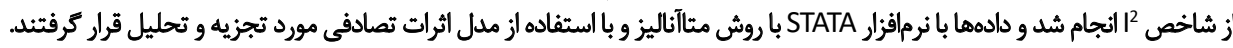

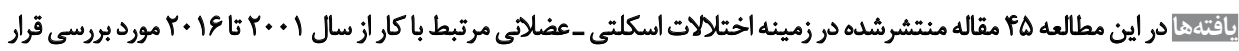

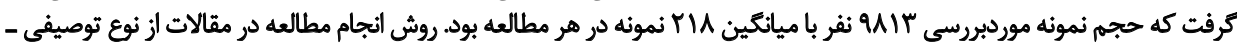

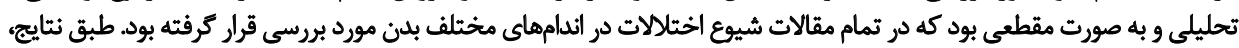

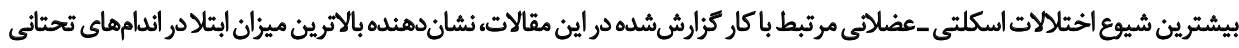

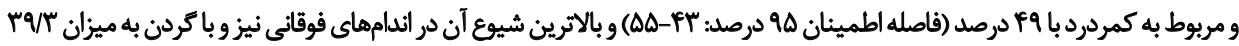

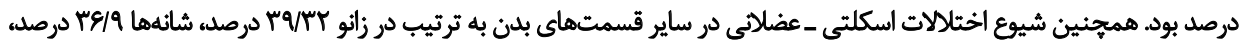

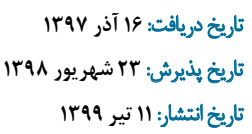

$$
\text { عليرضاخمر }
$$

نشانى: زابل، دانشكاه علوم يزتشكى زابل، كروه مهيندسى بهداشت حرفه اي،

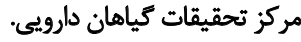

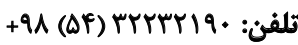
رايائامه: alireza.khammar@zbmu.ac.ir

$$
\begin{aligned}
& \text { * نويسئدكان مسئول: } \\
& \text { محسن يور صادقيان } \\
& \text { بهائتيني } \\
& \text { تلفن: }
\end{aligned}
$$
رايانامه: mo.poursadeghiyan@uswr.ac.ir 


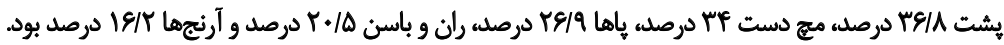

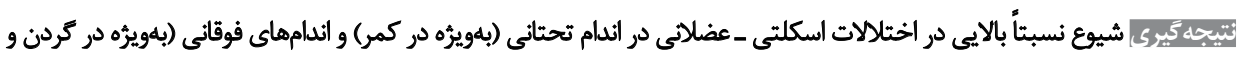

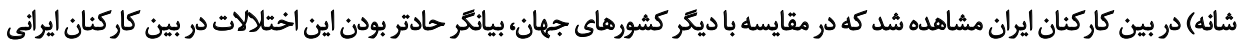

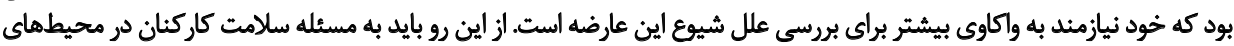

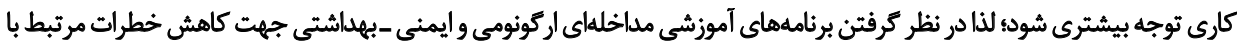

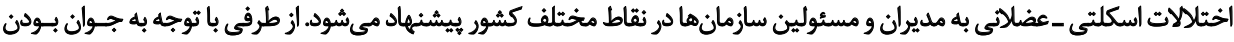

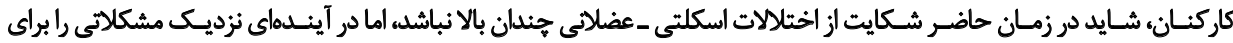

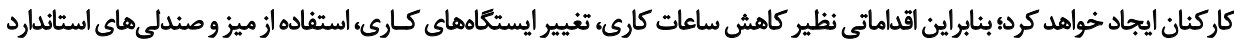

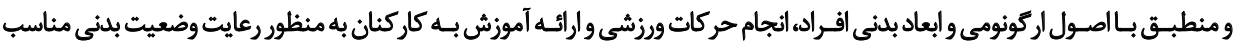

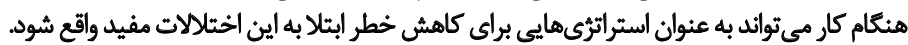

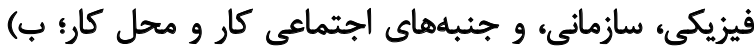
مElad

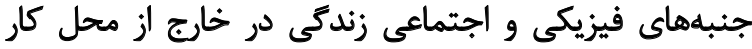

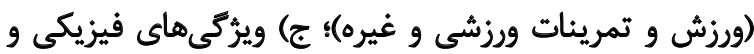
فيزيولوزيكى فرد اشاره كرد [عرينات ورشي فيره آسيبهاى اسكلتى ـ عضلانى ناشى از كار سبب تحميل هزينههاى زيادى به سيستم بهداشتى ـ ـ درمانى كشورها

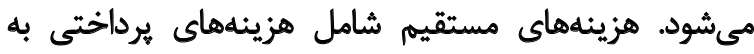

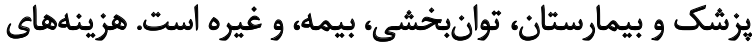

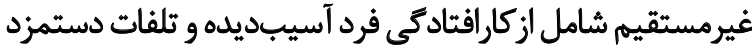

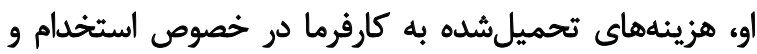

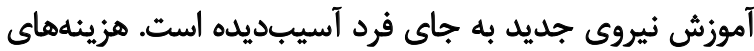

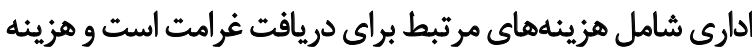

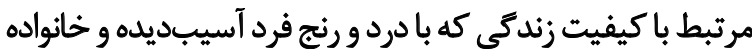

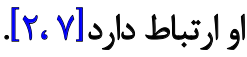

در كشور رائن رشد بيمارىهاي اسكلتى ـعضلانى در سال هاى

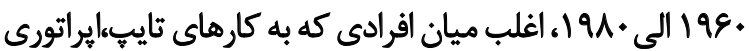

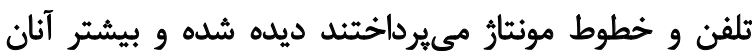

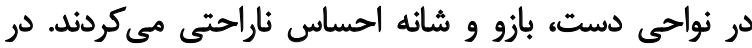

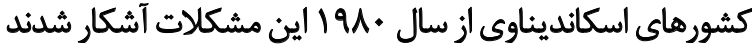

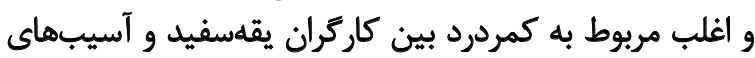

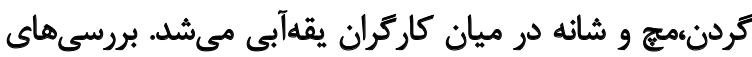

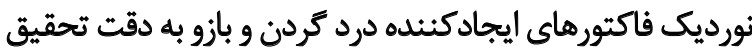

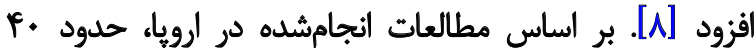

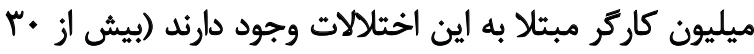

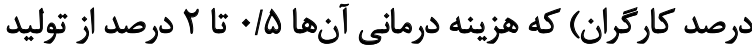

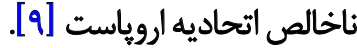
در ايران نيز مطالعات زيادى در اين رابطه انجام شده است

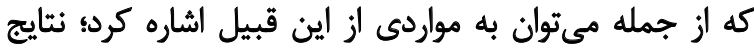

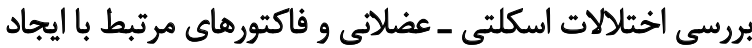

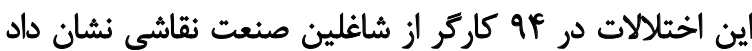

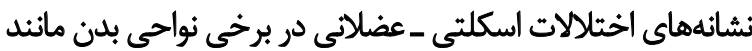

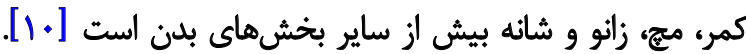

انستيتو ملى ايمنى و بهداشت شغلى آمريكا (NIOSH)

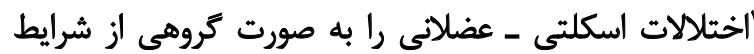

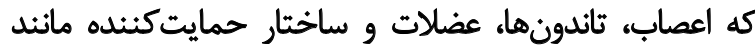

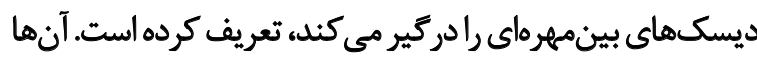

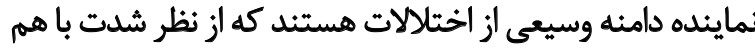

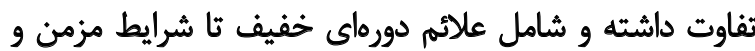

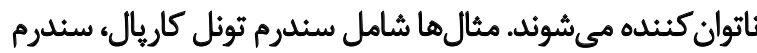

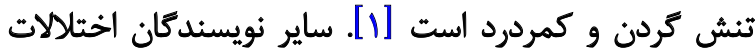

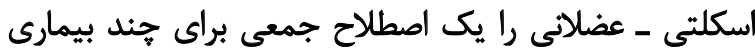

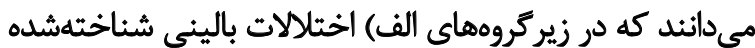

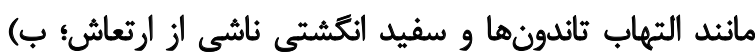

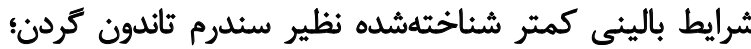

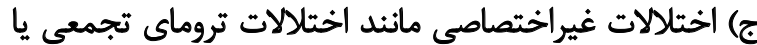

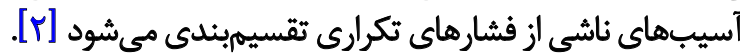

مسائل ايمنى و بهداشتى در محيطهاى شغلى از اهميت بسيارى

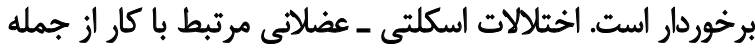

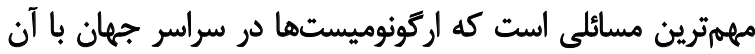

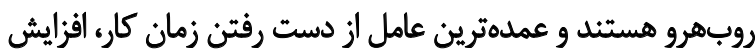

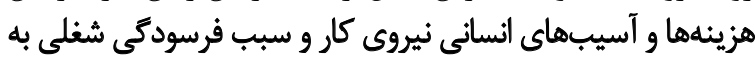

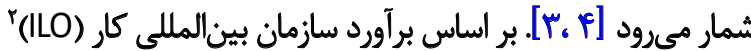

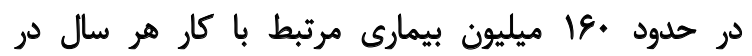

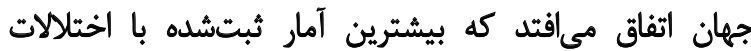

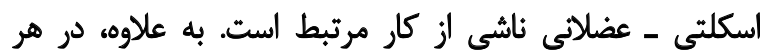

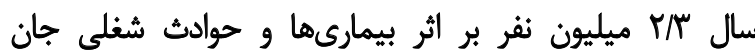

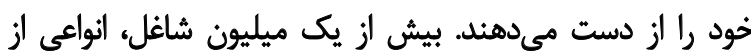

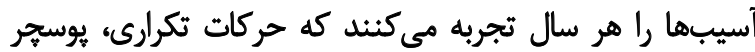

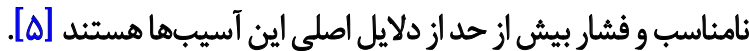

فاكتورهاى متعددى در ايجاد آسيبهاى اسكلتى ـ عضلانى

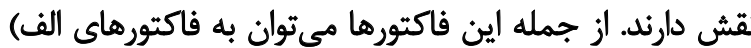

1. National Institution of Safety and Health

2. International Labor Organization 
عضلانى در دوره زماني يكساله، روش انجام مطالعه به شكل

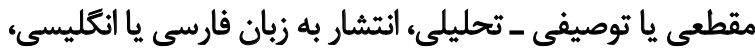

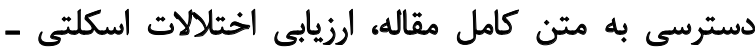

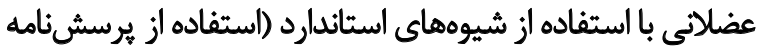
استاندارد اختلالات اسكلتى - عضلانى نورديك التفائ (NMQ) و در نظر كرفتن شيوع اختلالات اسكلتى ـ عضلانى تمام بدن إنان "اندامهاى فوقانى و تحتانى") بودئ

يرسشنامه نورديك يكى از معمولترين و استانداردترين

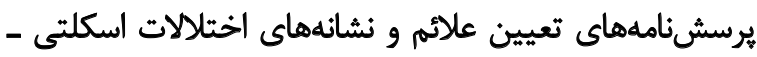

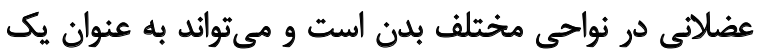

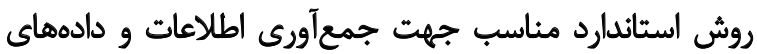

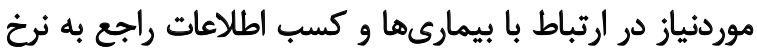

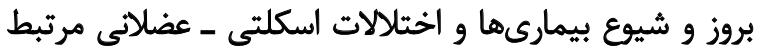

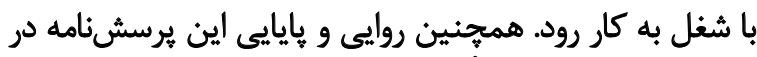
مطالعات مختلف مورد تأييد قرار كرفته است ورون

\section{انتخاب مطالعات و استخر إج دادهها}

درا ابتدا ليستى از عناوين و جإكيده كليه مقالات موجود در

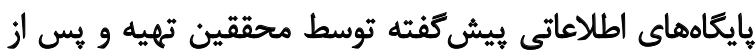

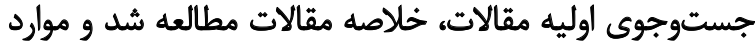

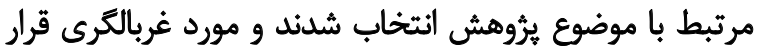

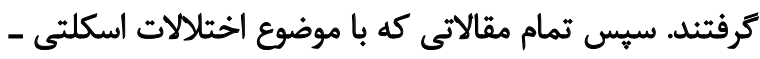

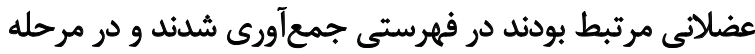

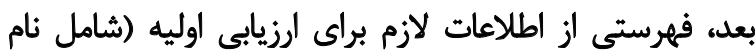

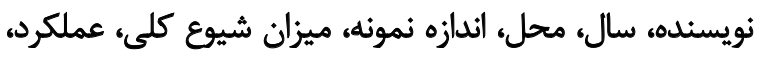

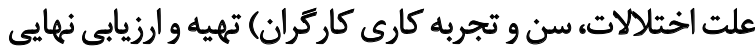
صورت ترفت و مقالات با عناوين تكرارى خارج شدارئد

در ادامه نيز جكيده مقالات به صورت دقيق براى يافتن مطالعات

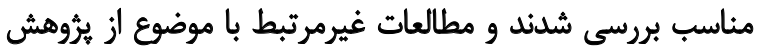

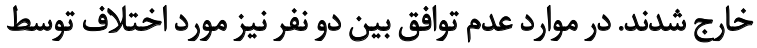

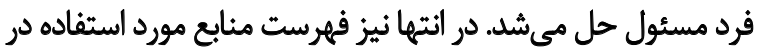

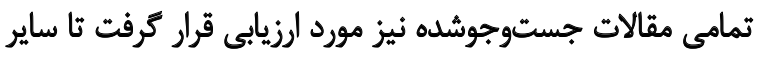

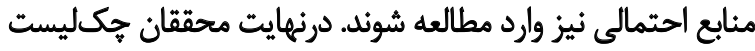

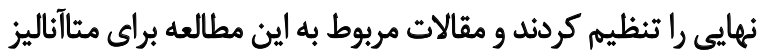

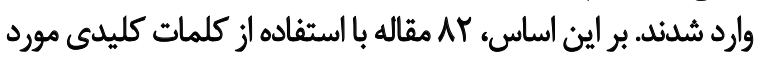

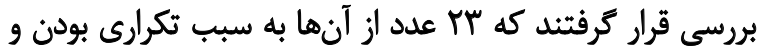

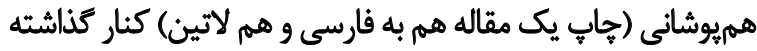

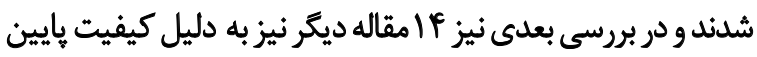

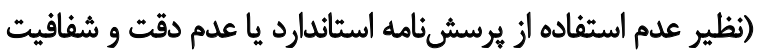

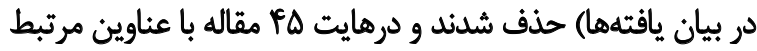

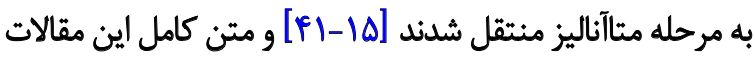

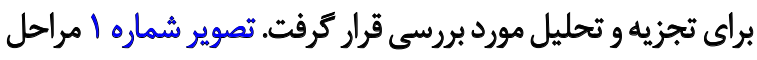
ورود مطالعات به مطالعه مثاآناليز رانشان مى قدهار ترفد.
همجينين نتايج مطالعه ارزيابى ريسك اركونومى و بررسى شيوع

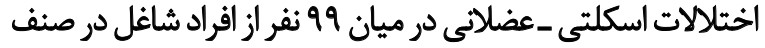

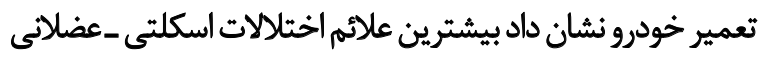

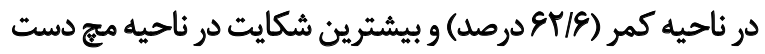

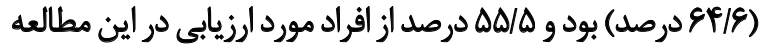

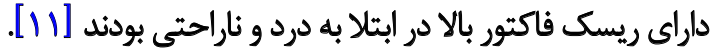

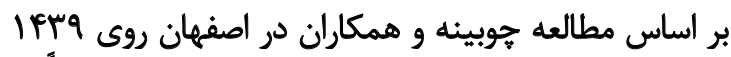

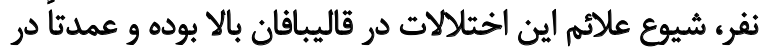

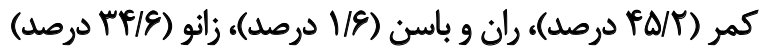

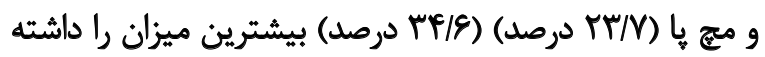

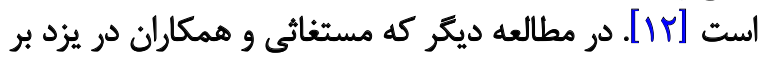

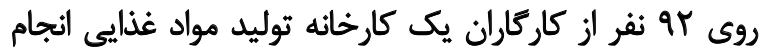

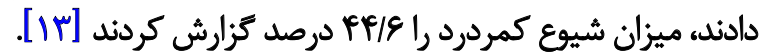

امروزه با توجه به اينكه بحث شيوع اختلالات اسكلتى - أندات

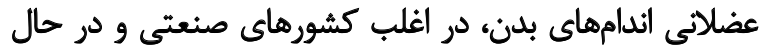

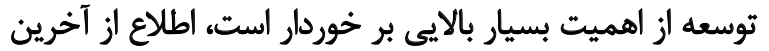

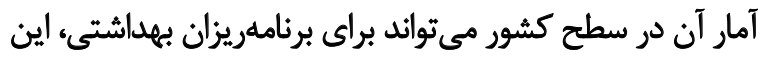

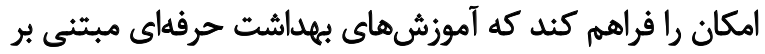

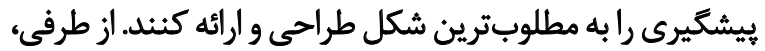

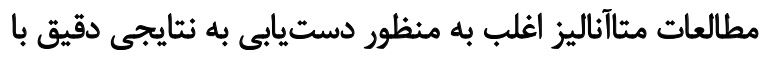

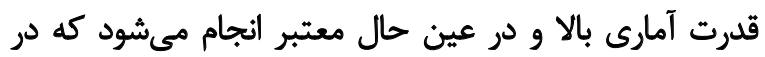

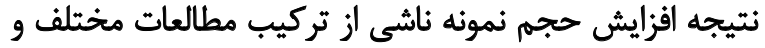

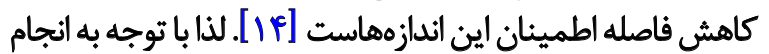

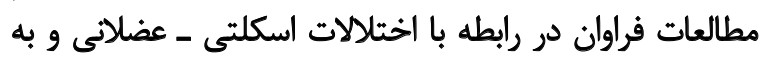

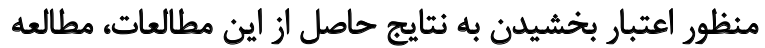

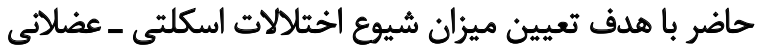

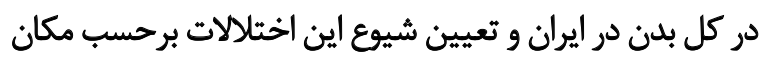

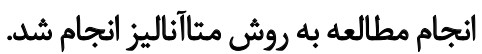

$$
\text { روش بررشى }
$$

مطالعه حاضر به صورت مطالعه فراتحليل (متآناليز) جهت إناتئ بررسى شيوع اختلالات اسكلتى ـ عضلاتى ناشى از كار در كشور

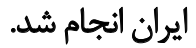

جهت جستوجوى مقالات مرتبط به دليل اينكه برخى

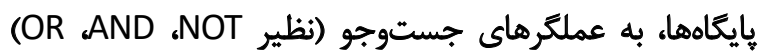

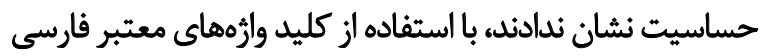

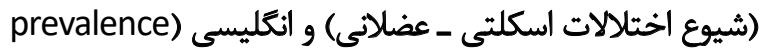

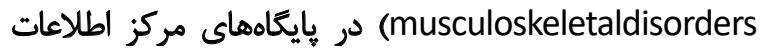

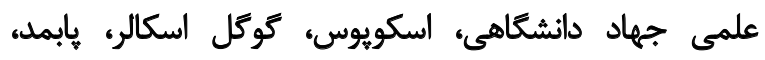

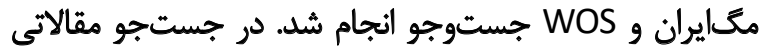

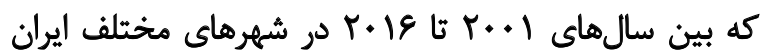

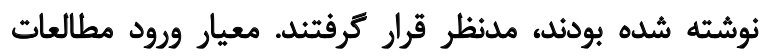
به بررسى، در نظر كرفتن ميزان شيوع اختلالات اسكلتى - مران 


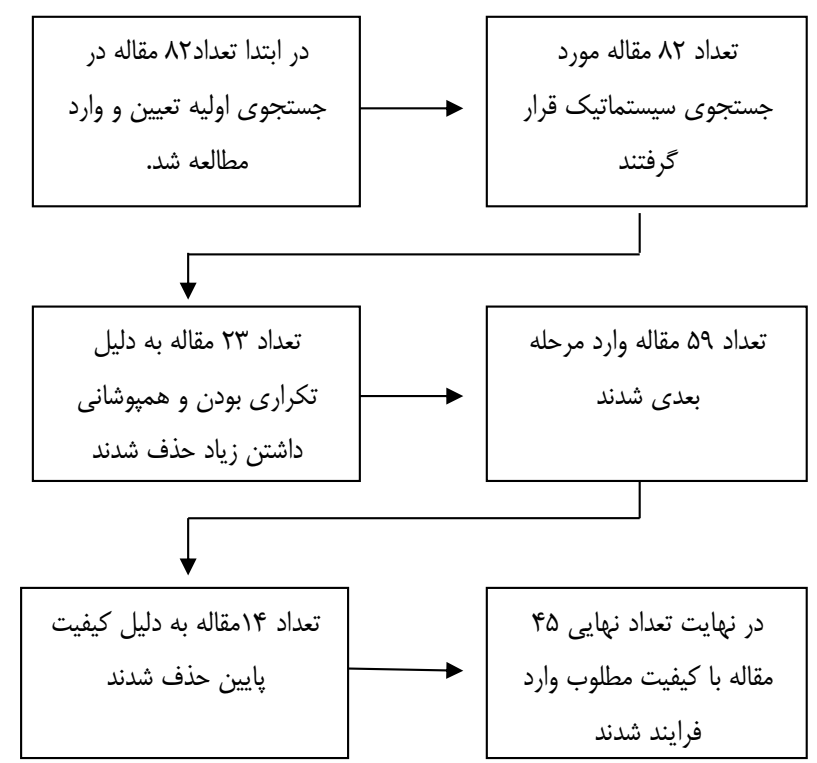

در اين مطالعه در جستوجوهاى اوليه AT مقاله شناسايى شد

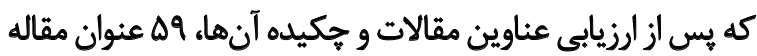

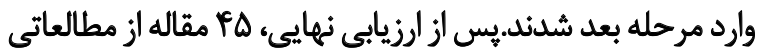

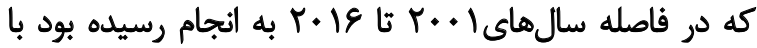

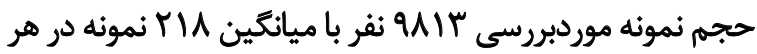

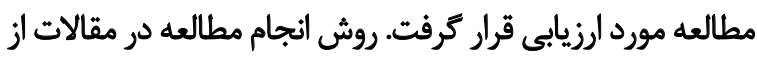

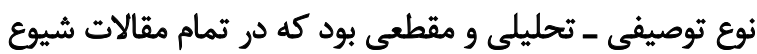

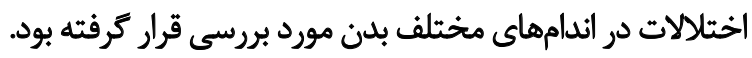

ميانكين سنى و سابقه كارى در اين مطالعات به ترتيب

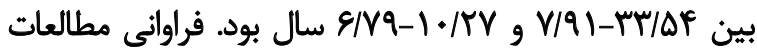

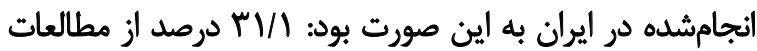

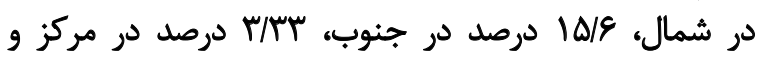

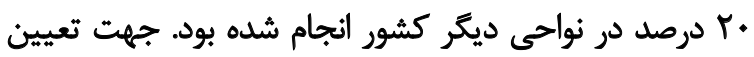

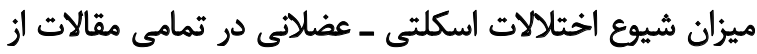

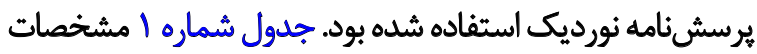

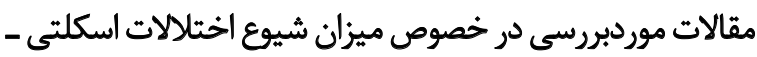

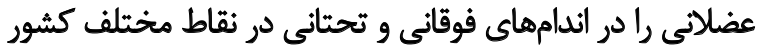

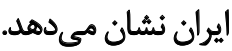

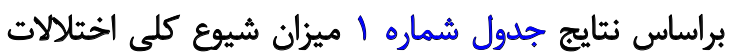

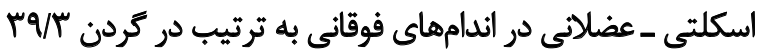

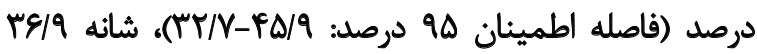

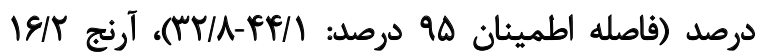

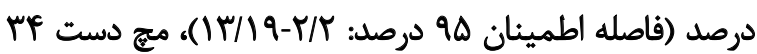

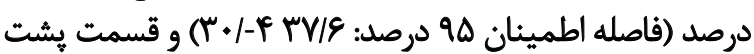

با توجه به اينكه در آناليز مطالعه، ميزان شيوع اختلالات

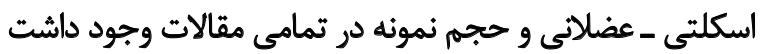

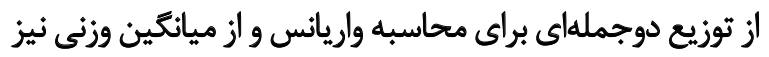
براى تركيب ميزان شيوع در مطالعات انجاميافته استفادياد شداند

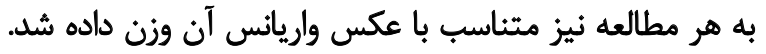

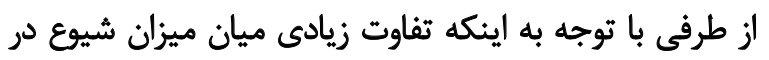

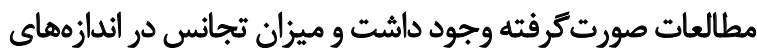
ميزان شيوع بيشتر از و

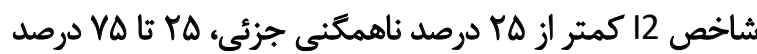

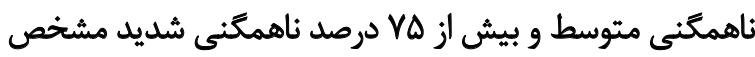

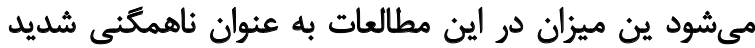
طبقلهبندى مىشود ين ميزان در در اين مطالعه از مدل اثرات تصادفى جهت برات بروسى بردها استفاده

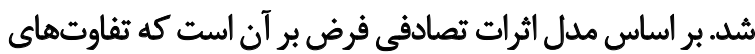

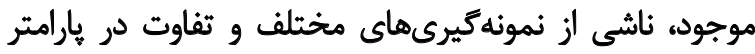

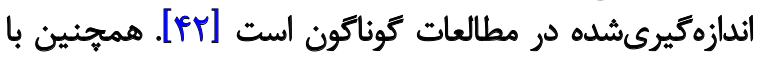

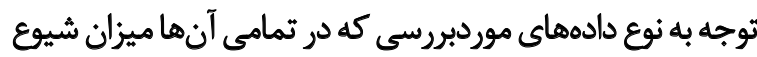

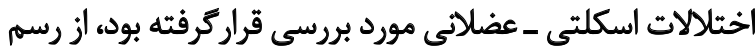

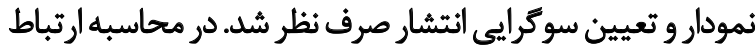

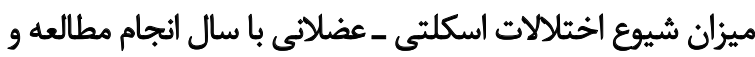

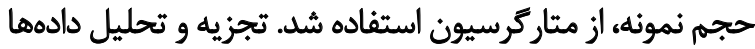
با استفاده از نرمافزار STATA (نسخه • (1) صورت بذئيرفت 
جدول ا. شيوع اختلالات اسكلتى ـ عضلانى در ايران

\begin{tabular}{|c|c|c|c|c|c|c|c|c|c|c|c|}
\hline \multicolumn{4}{|c|}{ درصد شيوع مشكلات اسكلتى ـ عضلانى در } & \multicolumn{4}{|c|}{ درصد شيوع مشكلات اسكلتى ـ عضلاتى در اندامهاى } & \multicolumn{2}{|c|}{ تعداد نمونه مطالعه } & \multirow{2}{*}{ سال انجام مطام } & \multirow{2}{*}{ 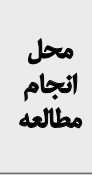 } \\
\hline بุ & زاتو & باسن و & كمر & آرنجّ & مئج دست & شائه & تردن & جدول(Y) & جدول(1) & & \\
\hline$\cdot \pi$ & - & .1 .9 & .1 .9 & - &.$/ T$ &.$/ \mathrm{Y}$ & $\cdot \pi r$ & M. & rr. & $r \cdot+r$ & تجبران \\
\hline- &.$/ M T$ & $\cdot / T \Delta \Delta$ &.$/ 411$ & $.1 \% 18$ &.$/ 481$ & . & . /AT & iv & ev & $r+\varphi^{f}$ & تهران \\
\hline.$/ T \omega$ & $.10 \cdot 4 \pi$ & t/IrIV &.$/ F a r$ & $+/ / F A$ & $\cdot / F$ & . $/ 4 \pi T$ & $. / R 8+1$ & 110 & 110 & $r+a$ & تهران \\
\hline $.1 . \Delta$ & $\% \Delta$ & & $.1 \Delta \cdot 1$ & - NIV &.$/ 450$ & - & -NEV & $r e$ & $r$ & $r .+1$ & كرمانشاه \\
\hline.$/ m n^{2}$ &.$/ p r$ &.$/ 1 Y$ &.$/ 4 \Lambda$ & . / IAf & $\cdot / \pi$ & $\cdot / \pi$ &.$/ M$ & r. & r. & $r+. q$ & تهران \\
\hline- & .1 .09$. & $\% 1+49$ & - & - & $.1+.9$ & $+1+49$ & $1 \cdot M$ & $1 \cdot r$ & $1 . r$ & $r+1$ & يزد | يزد \\
\hline- & - & - & - & $.1 . \Delta 1$ &.$/ N F$ & $\cdot / r \cdot r$ & - Mesa & $n$ & $n$ & r.oq & يزي \\
\hline- & - & - & - & & . MTA &.$/ 1 n$ & .1 .99 & 9r & १५ & r.l. & يزد \\
\hline 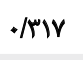 &.$/$ rap & . MIE & .latr &.$/ M A 9$ &.$/ F M$ &.$/ \Delta Q 8$ & IAAT & YM & $T M$ & r.. & شيراز \\
\hline.$/ T H Y$ &.$/ 491$ & . MTf &.$/ \pi n$ & $+/ \Delta$ &.$/ M r$ & $\cdot / M V$ & . Mat & rr & tr & $r+\infty$ & تهران \\
\hline .1 .11 & ATrV & $.1 .8 \mathrm{~V}$ &.$/ M P r$ & $1 .+\Delta$ & ./4TA & $-M+\varphi$ & - MAN & 19 & 19 & ro. & اروميه \\
\hline.$/ F \Delta \Delta$ & 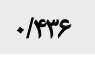 & $\cdot / \Delta \Delta \Delta$ & - & - & - & - & - & 11. & 111. & $r \cdot \cdot r$ & تكاب \\
\hline.$/ \Delta q$. & $. / \Delta 1 \mid$ & $+\pi+\gamma$ & . late & $+/ T Y q$ & $+/ F n$ & t/DIV & .1019 & Tro & rva & $r++Y$ & شيراز \\
\hline$\cdot / T H P$ & . & .1 .94 &.$/ 4 P 1$ & $.1 . v 8$ & ודוM. & 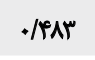 &.$/ Y A$ & ITD & lis & r..a & تهران \\
\hline.$/ M V$ & . Mes &.$/ 8$ &.$/ T W$ & - /19Y & . THAY &.$/ F r A$ & . THAT & 1199 & 1979 & r.+r & الصفهان \\
\hline $.1 .1 \Delta$ &.$/ 24$ &.$/ 1 Y A$ & - &.$/ 1 K A$ &.$/ 74$ & $\cdot / / Y$ & ./NFq & iv & pV & $r+\infty 1$ & قمم \\
\hline rIT & . MAT &.$/ 1 T$ &.$/ 148$ & $.1 \cdot \Delta F$ & . /TAF &.$/ \pi T r$ & .Mr & TEA & TFA & $r+1$ & قم \\
\hline - NAF & $-x+1$ & ./IMF & 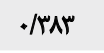 & ./NTA & $\cdot \pi \cdot \Delta$ & / TraF & $\cdot / K \Lambda$ & $\pi$ & $\pi$ & $r \ldots q$ & تهران \\
\hline . &.$/ 8 \pi$ & & - & - & - & - & - & $m$ & $M$ & $r++1$ & اراك \\
\hline.$/ m r^{2}$ &.$/ 40$ & $* / / \gamma$ & - & - & . $/ \pi \Delta$ & & $+\pi$ & FAF & PAF & $r+\infty$ & شيراز \\
\hline- & 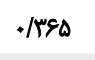 & & - & - &.$/ T 9 \Delta$ &.$/ \% \Delta$ & .10 & $p$ & P.. & r.aq & آمل \\
\hline / MY &.$/ P F$ &.$/ 19$. & שT/R & - & . & 年/ &.$/ 19$ & rep & res & $r . . r$ & سبزوار \\
\hline . & - & - & - & - &.$/ 198$ & +1199 & .1 .48 & rAf & rNF & $r+. q$ & كرمان \\
\hline- & - & - & - &.$/ I F A$ & . TTE &.$/ \% 19$ & - & af & $\Delta f$ & $r+. q$ & مييد \\
\hline.$/ N$ & .188 &.$/ F A$ & .1 .48 & $\cdot / r$ &.$/ \pi \Lambda$ &.$/ 44$ & $\cdot \pi F$ & $\Delta$. & a. & $r . . q$ & الصفهان \\
\hline .11 .9 & ./FTq & - &.$/ r I r$ & .1 .9 &.$/ T r$ & T &.$/ \pi I$ & 109 & 108 & $r++1$ & شيراز \\
\hline .119 & $+/ T \Delta 1$ & . MV & . & . IFT & . I8W & $+/ M V$ & $\cdot M r$ & vo & va & $r+\infty$ & شيراز \\
\hline.$|m|$ & r هq, & $+N+P$ &.$M T$ &.$/ r n$ & . & /ATV & . $10 \mathrm{rr}$ & $\Delta \varphi$ & $\Delta r$ & $r \cdot I r$ & قزوين \\
\hline- & - & - & - & . If & $\cdot / \pi \cdot 9$ &.$/ 79 \Delta$ & IAIF & Mr. & $\pi$. & $r \cdot 1 r$ & يزد \\
\hline .1 .90 &.$/ T V$ &.$/ 11 F$ & . IAT & $* \%$ &.$/ 19$ & . TET & TH & F.. & $r .$. & $r \cdot I r$ & رفسنجان \\
\hline- &. $\mid N P$ & - & . & .1 .14 &.$/ 2 \mathrm{ra}$ & . $/$ rag & - /par & $n$ & $n$ & $r+11$ & همبان \\
\hline- & - & - & .1 .19 & - & - & - & . MAF & ef & 9 & $r .1 f$ & بايل \\
\hline .11. & $* \pi+$. & - &.$/ \%$ & $1.8 V$ & $.11+$. & "זr/. & . & r. & r. & $r+1 f$ & هملان \\
\hline- &.$/ 118$ & - & $. / r+F$ & - & - & - &.$/ 481$ & Irk & IrA & $r+18$ & الصفهان \\
\hline . &.$/ m e r$ & .IVE & $\cdot /$ Tr & MFA &.$/ M T H$ & . $M Y$ & .1097 & ive & ive & $r+1 r$ & تيثران \\
\hline •/rAY &.$/ r r$ &.$/ 119$ &.$/ 199$ & . IIfe &.$/ \pi$ & . / & $.10 \cdot \Delta$ & 9f. & १९. & $r+10$ & سارى \\
\hline
\end{tabular}




\begin{tabular}{|c|c|c|c|c|c|c|c|c|c|c|c|}
\hline \multicolumn{4}{|c|}{ درصد شيوع مشكلات اسكلتى ـ عضلاتى در } & \multicolumn{4}{|c|}{ درصد شيوع مشكلات اسكلتى ـ عضلاتى در اندامهاى } & \multicolumn{2}{|c|}{ تعداد نموثه مطالعه } & \multirow{2}{*}{ سال انجام } & \multirow{2}{*}{ مطالع محلم } \\
\hline مج ها & زانو & باسن و & كمر & أرنج & ميج دست & شاثه & تردن & جدول(r) & جدول(1) & & \\
\hline.$/ N F$ & $\cdot / \pi$ & |/ & |r|r|r & $1 . r r$ &.$/ 1 Q A$ &.$/ A T T$ & $. / \Delta \mid F$ & MTr & MH & $r \cdot 10$ & تكاب \\
\hline . $/ \pi T$ &.$/ \$ \& A$ & - / IOY & . /TAq &.$/ 1 n$ & . & . /TAY & . MTE & rof & rof & $r \cdot 10$ & ايران \\
\hline . $R \varepsilon_{0}$ & . & - &.$/ 9 T F$ & $\cdot / r \cdot$. & $.1 \%$ &.$/ 4 q$ &.$/ M M$ & Yo. & TA. & $r \cdot 1 r$ & سنئدج \\
\hline- &.$/ 8 \mathrm{~V}$ & - & - & - &.$/ 2 q$. &.$/ T \wedge$ & . $/ \Delta H^{\circ}$ & re & re & $r \cdot 1 r$ & ك \\
\hline \% &.$/ 19 \Delta$ & $\cdot 11 \cdot 1$ & - & - &.$/ T \Delta$ &.$/ T \Delta$ &.$/ \pi T$ & QT & 95 & $r \cdot 11$ & كيلان \\
\hline . & . IPIA & $1 \cdot W$ &.$M A$ & . IT & - TAS & - THar & - Far & 91 & 91 & $r \cdot 1 r$ & اصفهان \\
\hline . & - $/ 419$ &.$/ N V$ & $.10 \pi T$ &.$/ T w$ & . $10 \Delta T$ &.$/ F 19$ & - /VAV & r.. & $r+$. & $r \cdot 1 r$ & تبريز \\
\hline$+/ r \Delta+$ & $t / F \Delta$ & . TE &.$/ \pi$ & $t / \pi$ & $* / T$ & $* / \pi$ & $+/ \pi$ & $1 .+$ & 1.0 & $r \cdot 1 r$ & سارى \\
\hline .1098 & - IraA &.$/ T q 1$ & $. / \Delta)^{2}$ & $.11 D A$ &.$/ P A 1$ & - $/ r \Delta \Delta$ &.$/ P N$ & TAD & TAD & $r \cdot I r$ & ايران \\
\hline
\end{tabular}

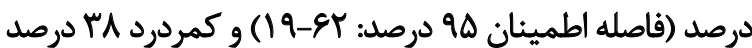

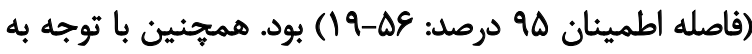

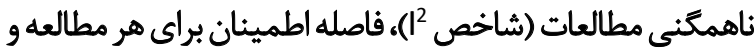

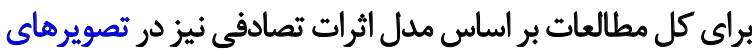

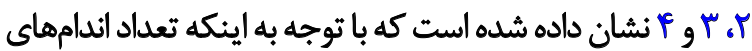

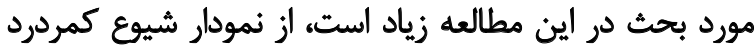

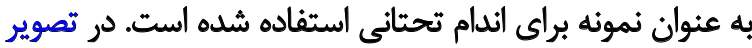

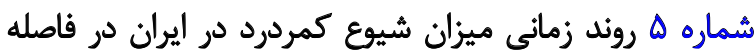

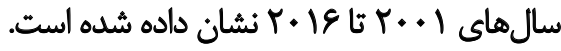

در تصوير شماره \& نمودار شيوع كمردرد به عنوان نمونه براى اندام تحتانى استفاده شده است. در اين نمودار ميزان شيوع

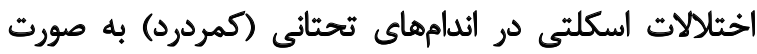

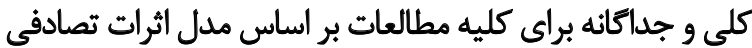

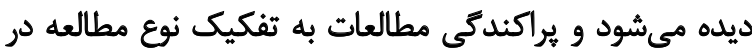

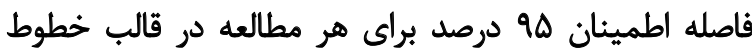

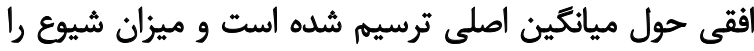

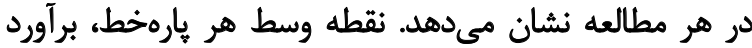

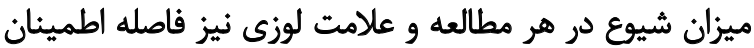

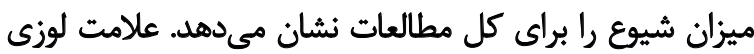

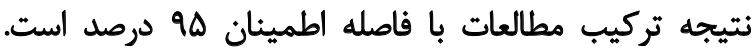

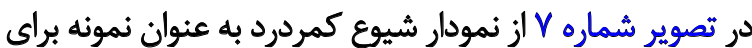
اندام تحتائى استفاده شده است.

در اين نمودار ميزان شيوع كمردرد به صورت كلي و جداكانه

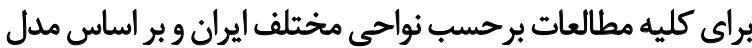

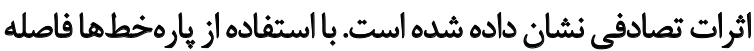

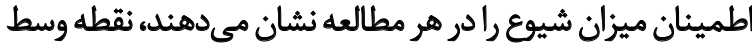

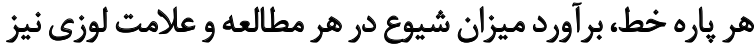

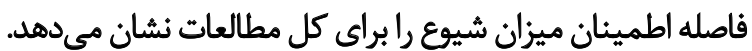

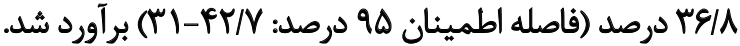
همجينين ميزان شيوع كلى اختلالات اسكلتى ـ عضلانى در

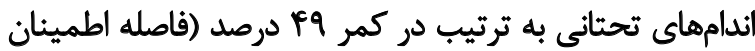

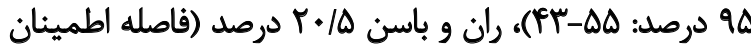
ه 9 درصد: درصد:

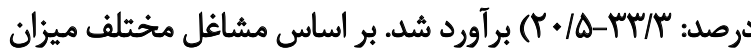

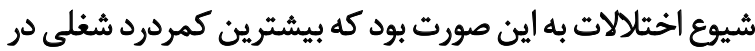

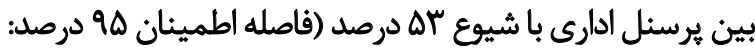

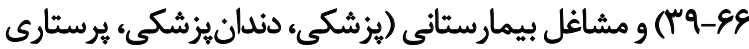

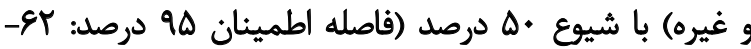

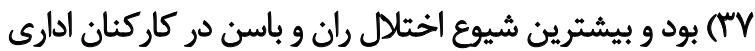

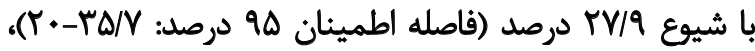

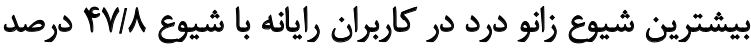

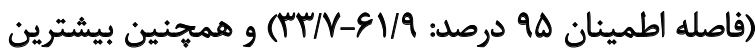

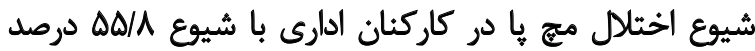

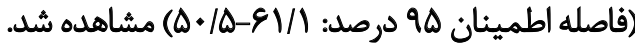

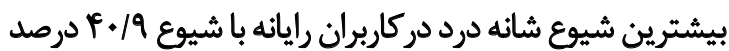

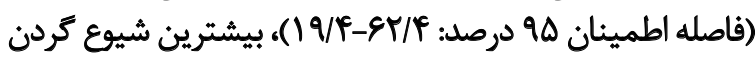

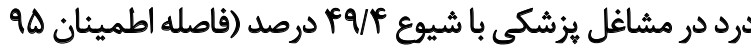

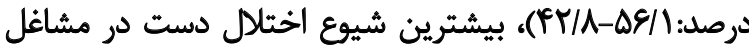

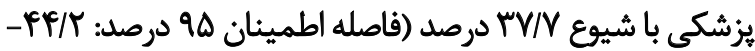

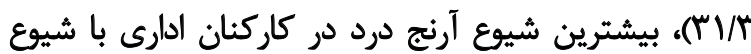

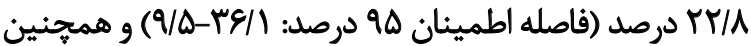
بيشترين شيوع خشت درد دركاربران رايانه با شيوع (فاصله

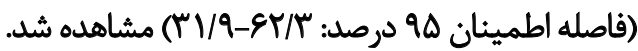

مطابق با يافتههاى جدول شماره ؟، ميزان شيوع اختلالات

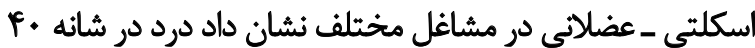


جدول Y. ميزان شيوع اختلالات اسكلتى ـ عضلانى بر اساس مشاغل مختلف

\begin{tabular}{|c|c|c|c|c|c|}
\hline كاركنان ادارى & كارئران صنايع دستى & كاريران در صنعت & كاركنان بيمارستان & كاربران راياته & \\
\hline$\cdot / \Delta \omega^{\prime}(\cdot N \varepsilon, \cdot N \cdot)$ &.$/ M a(\cdot / \pi, \cdot / \Delta \Delta)$ & $\circ / \Gamma \Lambda(\cdot / / \gamma, \cdot / \kappa \Lambda)$ &.$/ 29(+/ R T, \cdot / \Delta S)$ & $\cdot / \pi T\left(\cdot / \Omega \Delta_{*} \cdot N \cdot\right)$ & كرن \\
\hline$\left.\cdot / \varphi \cdot(\cdot / T \Delta, \cdot / \Delta)^{\circ}\right)$ & $\cdot R A\left(\cdot / T q, \cdot / N^{*} A\right)$ & $\cdot \pi\left(\cdot / \Lambda_{0} \cdot R_{*}\right)$ & $\cdot M V\left(\cdot M_{*}, \cdot N^{*}\right)$ & $\cdot / 4 \cdot(\cdot / 19, \cdot / P Y)$ & شائه \\
\hline$\cdot / \pi r(\cdot 1 \cdot q, \cdot \pi r)$ & $\cdot N \Delta(\cdot 1 \cdot \Lambda, \cdot N M)$ & $\cdot / \Delta \Delta(\cdot N \cdot, \cdot / r \cdot)$ &.$/ N P(\cdot / \cdot V, \cdot / T Y)$ &.$N E(\cdot / * r, \cdot N+)$ & آرنغ \\
\hline$\cdot \pi /(\cdot / T, \cdot / \Delta \cdot)$ & $\cdot / N \Delta(\cdot / T \xi, \cdot / \mu \varphi)$ & 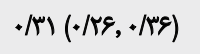 & $\cdot / \pi r(\cdot / \pi), \cdot / \pi r)$ &.$/ M 1(\cdot / N \cdot, \cdot(\Delta))$ & هيج دست و دست \\
\hline$\cdot / \pi \Lambda(\cdot / 1 q, \cdot / \Delta C)$ & $. / F)(. / T Y, \cdot / \Delta E)$ & $\cdot / \pi \cdot(\cdot / M, \cdot / N \Lambda)$ &.$/ 29(\cdot / T \&, \cdot / \Delta Y)$ & $\cdot \operatorname{ler}(\cdot / \pi), \cdot / 8 T)$ & ليشت \\
\hline$\cdot / \Delta T(\cdot / M q, \cdot / 89)$ & $\cdot / F q(\cdot / F T, \cdot / \Delta V)$ & $\cdot / * q(\cdot A r, \cdot / 8))$ & $\cdot / \Delta \cdot(\cdot / \pi Y, \cdot / 8 T)$ & $\cdot / \pi T(\cdot / R E, \cdot / \Delta A)$ & ل يشت كمر \\
\hline$* / M(* / \cdot 9, \cdot / M)$ &.$/ N \Delta(\cdot / N F, * / N Y)$ & $\cdot / M\left(\cdot / N r_{2} \cdot / T^{\prime}\right)$ & $\cdot / r V\left(\cdot / T_{*}, \cdot / \pi \Delta\right)$ &.$/ 19(\cdot / 18, \cdot / \pi)$ & رانها و باسن (باسن) \\
\hline.$/ F r(\cdot / \pi r, \cdot / 81)$ & $\cdot / r \Delta(\cdot / r q, \cdot / F u)$ & $\cdot / T V(\cdot / T V, \cdot / F \lambda)$ & $\cdot / \pi \gamma(\cdot / \pi \varepsilon, \cdot / \pi N)$ & $\cdot / M Y(\cdot / M T, \cdot / T Y)$ & 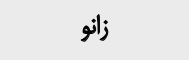 \\
\hline$+/ T Y(\cdot / \cdot A,+/ F \Delta)$ & $\cdot / T \&(\cdot / N Q, \cdot / T Y)$ & $+/ 19(* / 11,+/ T 1)$ &.$/ \pi Y(+/ I Y, \cdot / N Y)$ & $\cdot / T Y(+/ T Y, \cdot / T E)$ & 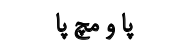 \\
\hline
\end{tabular}

اساس مدل اثرات تصادفي نشان داده شده است. يارهخط هذا فاصله

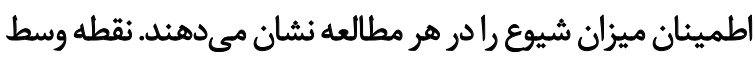

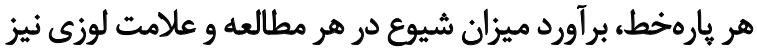

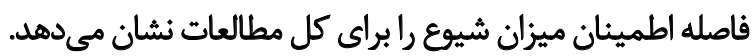

در تصوير شماره 1 از نمودار شيوع كمردرد به عنوان نمونه

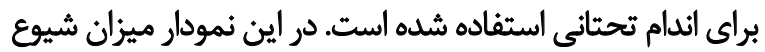

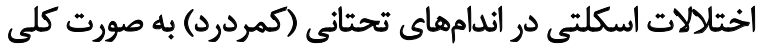

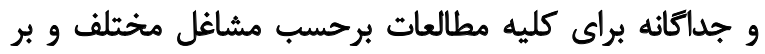

Study
ID

Gharagozlo $F(2001)$

Chobine A (2003)
Mir Mohammadi M (2003)

Mir Mohammadi M
Saremi M (2004)
Chobine A (2005)

Koshtkaran A (2005)
Ghasem Khani M (2005)

Nass seraij G (2006)

Chobine A (2006)

Nasi seraji G (2007)

Razavi S (2007)

Halvani G (2008)

Ghamari $F(2008)$

Chobine A (2008)

Chobine A (2008)

Chobine A (2008)

Sharifnia H (2009)

Mohammad Fam E (2009)

GHarib S (2009)

Mehrparvar A (2009)
Hashemi Negad N (2009)

Hashemi Negad N (2009)
Shanba M (2009)

Shanba M (2009)

Motalebi Kashani M
Mostaghaei M (2010)

Ahmadi F (2011)
Rabiei M (2011)

Mehdi pour H (2012)

Gorgi Z (2012)

Mosavi fard A (2012)

Nazari $\mathrm{j}(2012)$

Choobineh A (2012)

Solhi M (2013)

Sharafi N (2013)

Loghmani A (2013)

Etemadinezhad S (2013)

Saifi S (2014)

aroni zadeh Z (2014)

Nasiri D (2015)

Ghaiybi L (2015)

Overall (I-squared $=97.1 \%, p=0.000$ )

NOTE: Weights are from random effects analysis

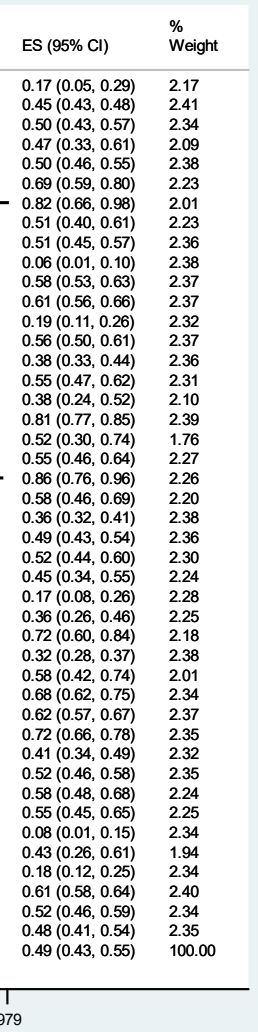

تصوير Y. براكيش مطالعات اسكلتى عضلاتى در ايران 
Study
ID

Central Iran

Chobine A (2003)
Nasl seraji G (2007)

Naslvani G (2008)
Ghamari F (2008)

Chobine A (2008)

GHarib S (2009)

Mehrparvar A (2009)

Mostaghaei M (2010)

Mehdi pour $\mathrm{H}(2012)$

Loghmani A (2013)

Motamedi M (2015)

Ghaiybi $\mathrm{L}(2015)$
Subtotal (l-squared $=98.0 \%, \mathrm{p}=0.000)$

North of Iran

North of Iran
Mir Mohammadi M (2003)

Saremi M (2004)

Ghasem Khani M (200)
Sharifnia H (2009)

Sharifnia $H(2009)$
Dehghani $Y(2009)$

Mohammad Fam E (2009)

Shanba M (2009)
Motalebi Kashani M (2009)

Rabiei M (2011)

Mosavi fard A (2012)

Solhi M (2013)

Saif S (2014)

Saifi S (2014)
Nasiri D (2015)

Subtotal (I-squared $=97.0 \%, p=0.000$ )

South of Iran

Chobine A (2005)

Chobine A (2006)

Chobine A (2007)
Chobine A (2008)

Hashemi Negad N (2009)

Gorgi Z (2012)

East of Iran

Razavi S (2007)
Subtotal (l-squared $=. \%, p=$.

West of Iran

West of Iran
Gharagozlo $F(2001)$
Nast seraij $G$ (2006)

Nasl seraji G (2006)
Ahmadi F (2011)

Nazari $i$ (2012)

Sharafi N (2013)

Subtotal (l-squared $=95.6 \%, p=0.000$ )

6

Choobineh A (2012)
Abdolahpour N (2015)

Abdolahpour $\mathrm{N}(2015)$
Subtotal (l-squared $=92.1 \%, \mathrm{p}=0.000$ )

Overall (I-squared $=97.1 \%, p=0.000$ )

NOTE: Weights are from random effects analysi

1
.979

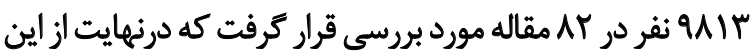

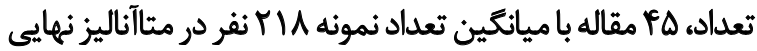

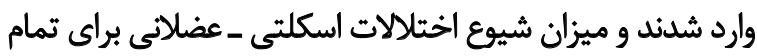

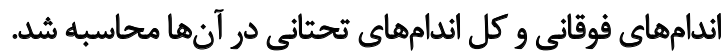
نتايج اين مطالعه حاكى از بالا بودن شيوع اين اختلالات در دري

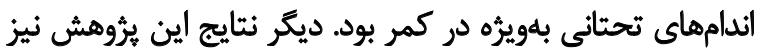

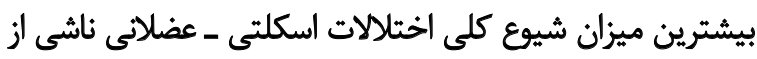

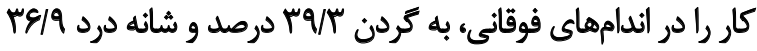

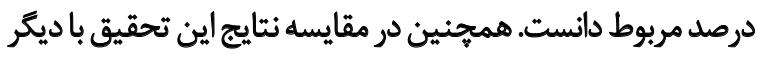

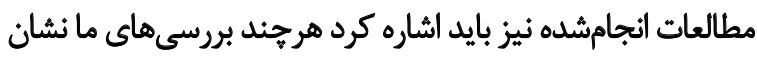

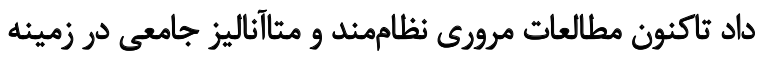

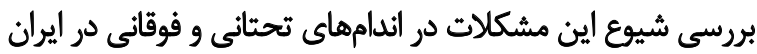

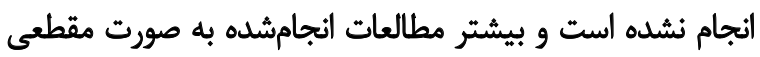

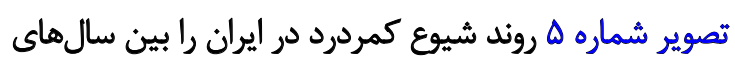

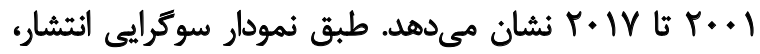

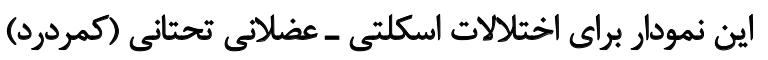
معنى اين نموار يريست تصوير شماره V، نمودار متاركرسيون برحسب ميانكين سابقه

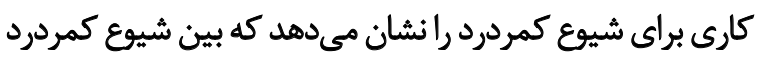

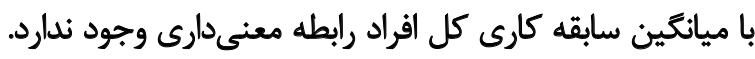

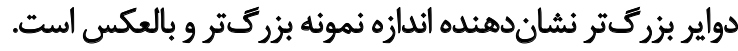

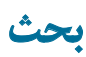

در مطالعه فراتحليل حاضر ميزان شيوع مشكلات اسكلتى - ماني عضلانى ناشى از كار در اندام تحتانى و فوقانى كارع ماركنان در بين التين 


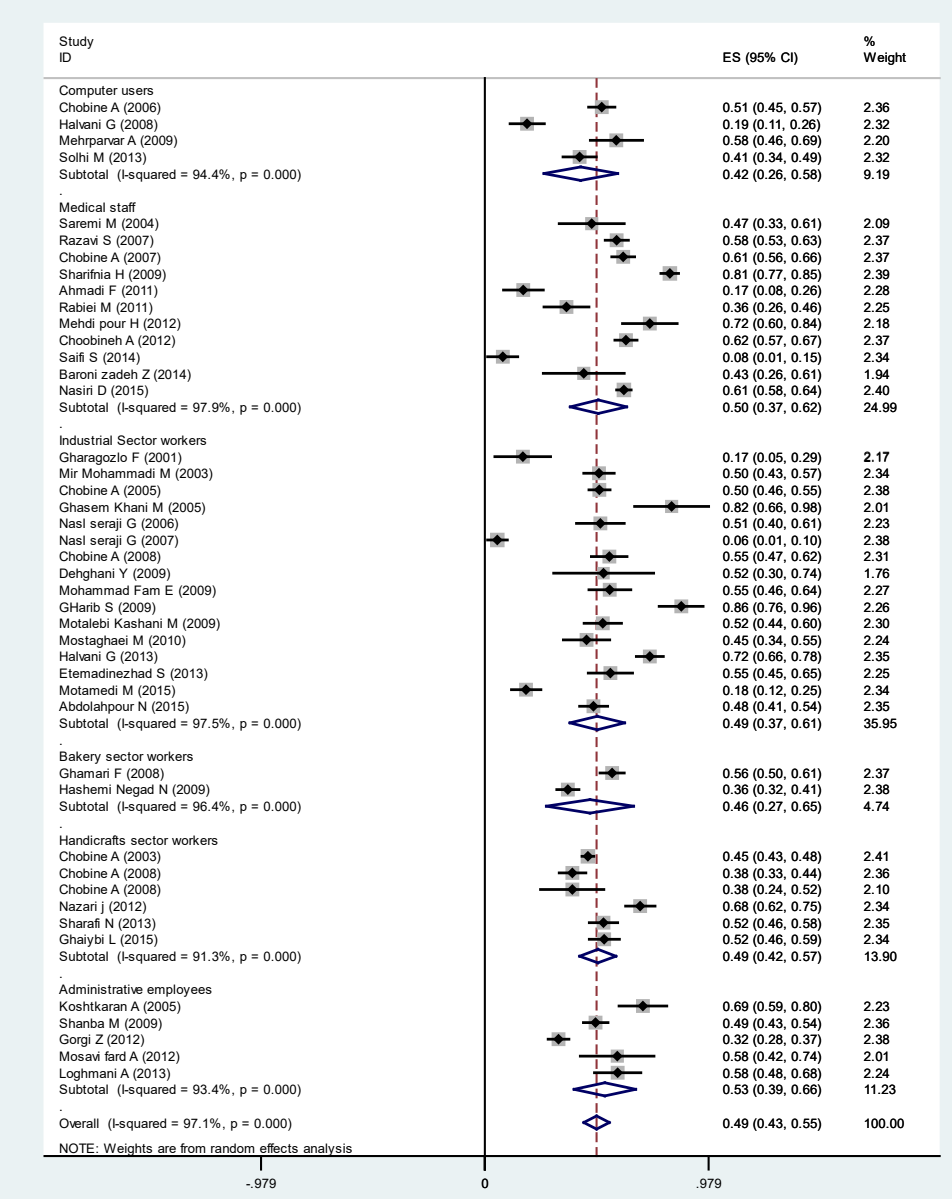

و يراكنش اختلالات بالهيكيلتيني

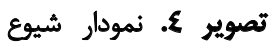

عضلانى در ايران

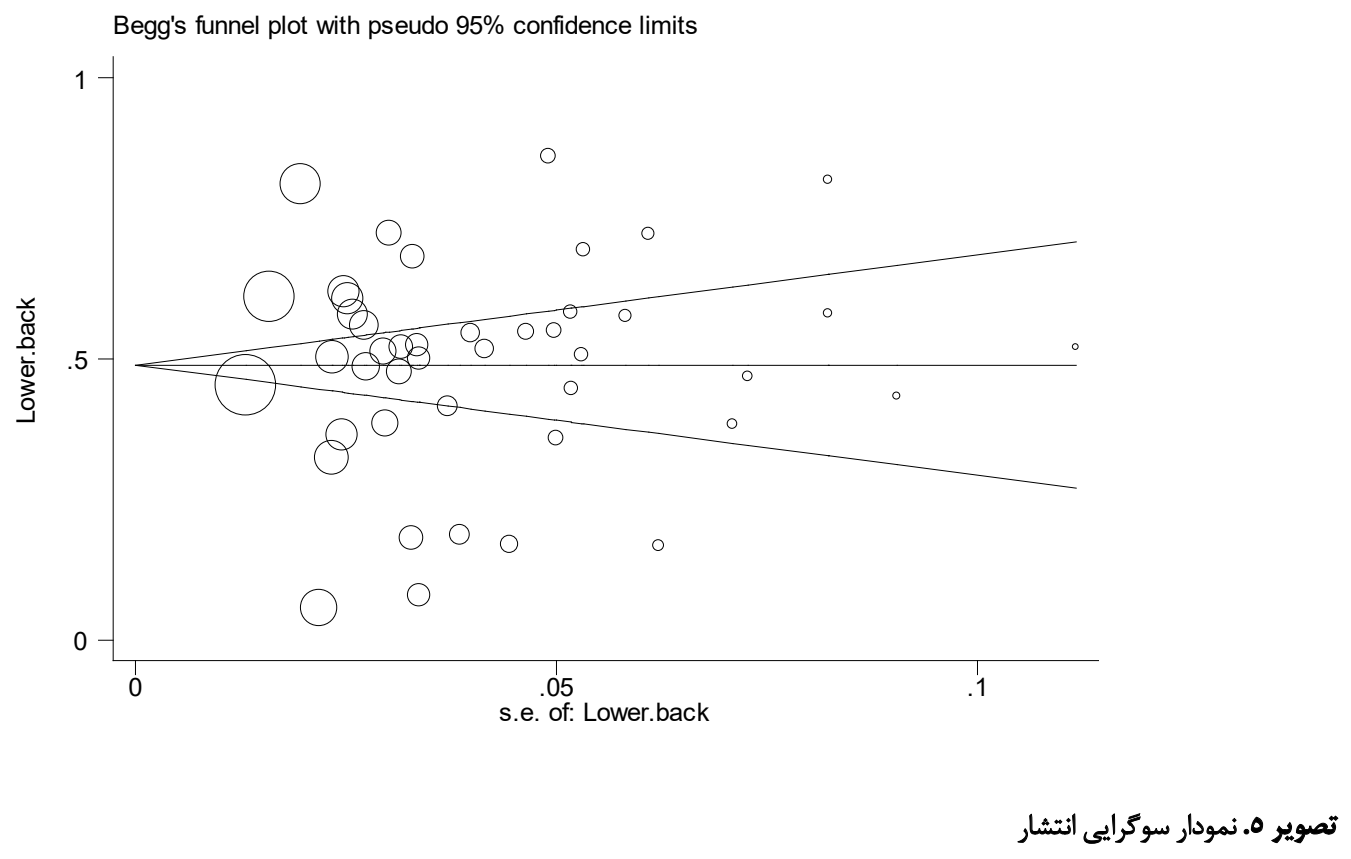




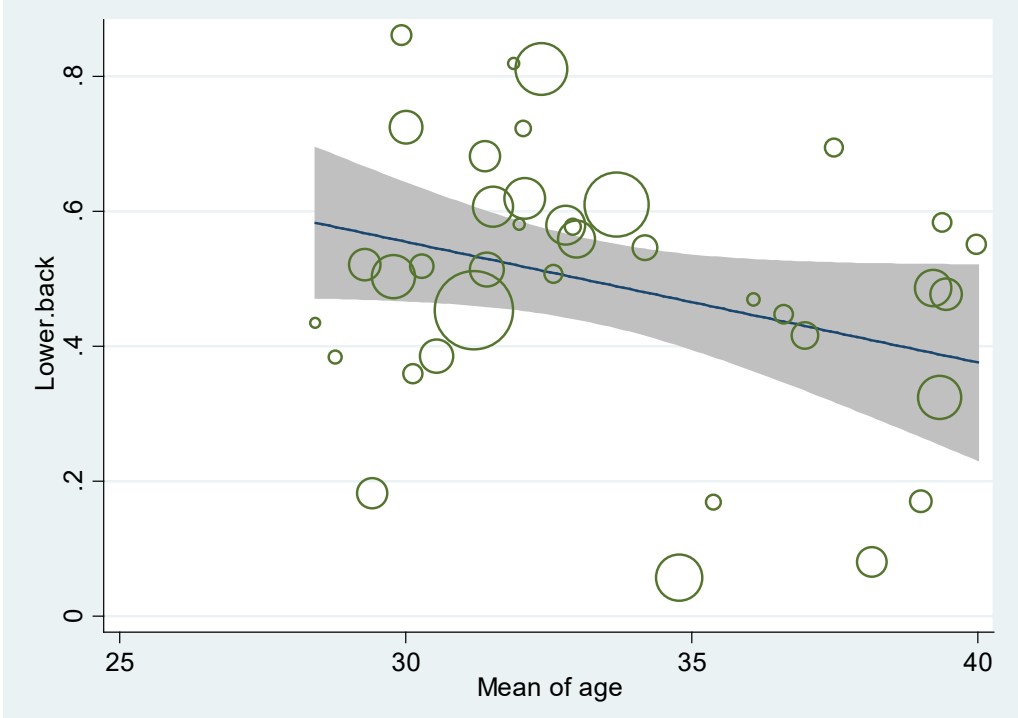

توانبخننى

تصوير 7. مطابق با نمودار متا ركرسيون فوق بين شيوع اختلالات عضلانى اسكلتى اندامهاى تحتانى (كمردرد) و ميانتين سن كل افراد رابطه معنى دارى وجود ندارد.

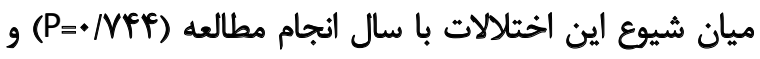

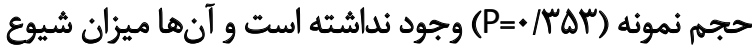

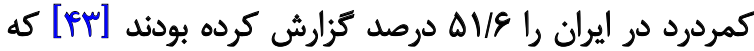

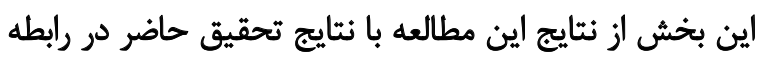

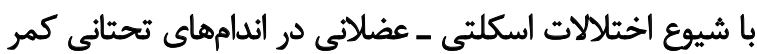

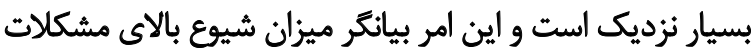

بوده است، اما مى توان به برخى از تحقيقات مرتبط از جمله مطالعه

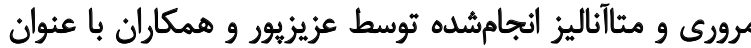

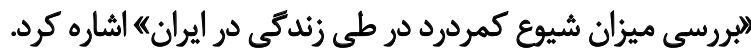

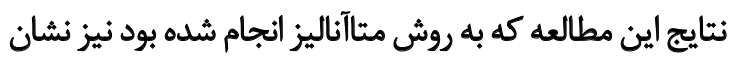

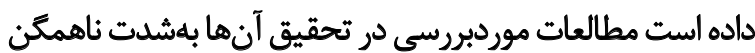

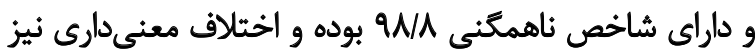

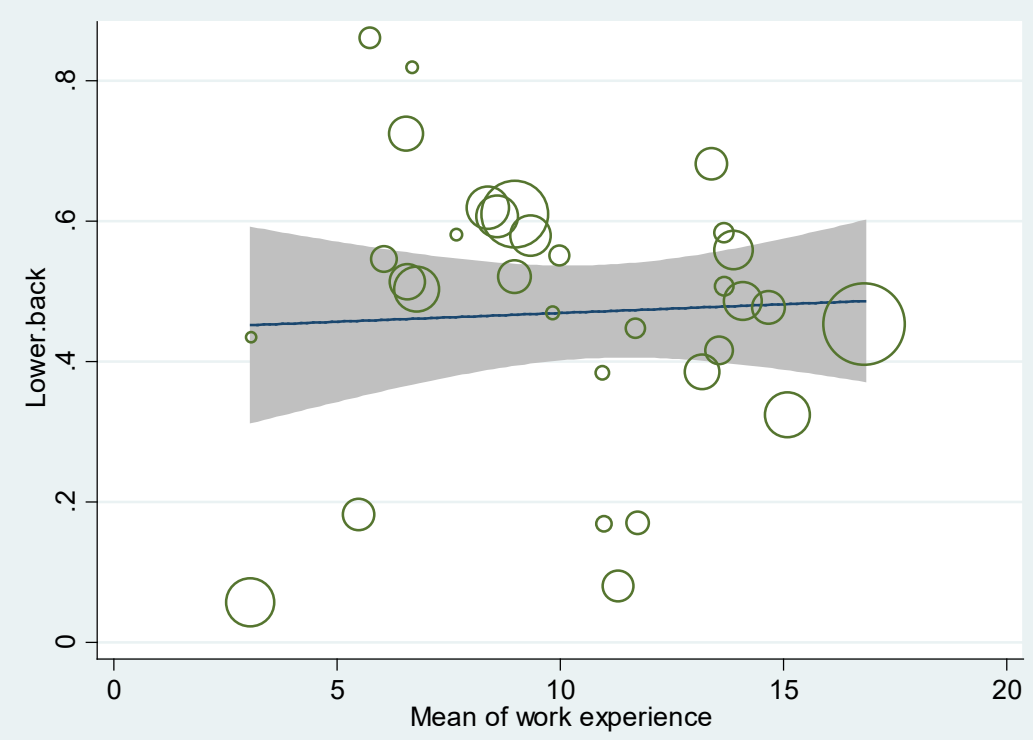

توانبخننى

تصوير لا. مطايق با نمودار مثاكرسيون فوق بين شيوع اختلالات اسكلتي ـ عضلاني اندامهاى تحتاني (كمردرد) و ميانكين سابقه كارى كل افراد رابطه معنى دارى 


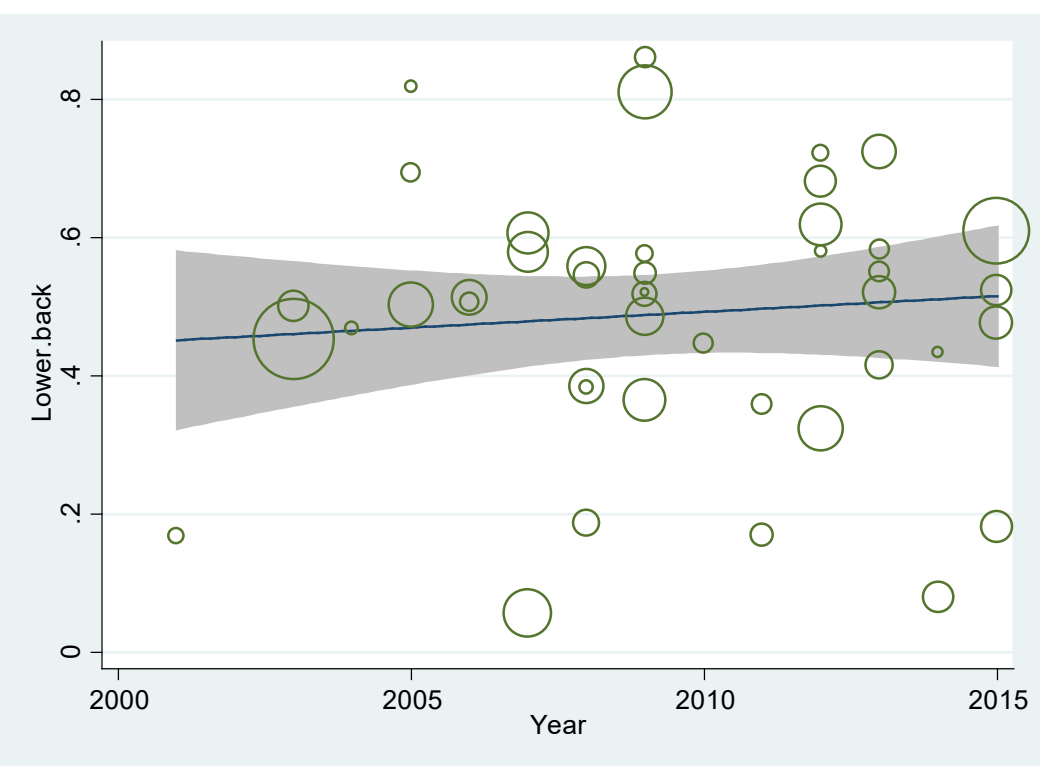

توانبخنتى

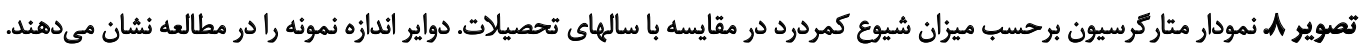

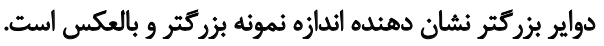

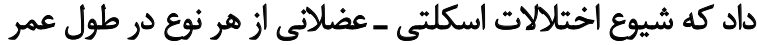
كشاورزان

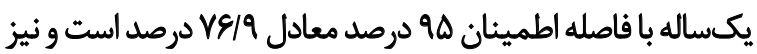

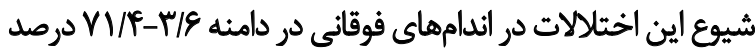

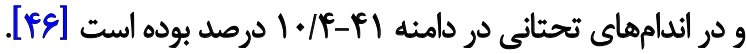

اين در حالى است كه در مطالعه ما نيز در ميان اختلالات

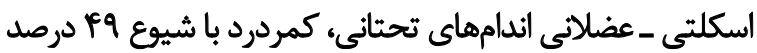

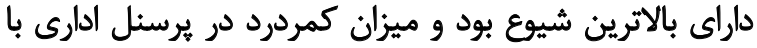

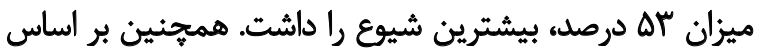

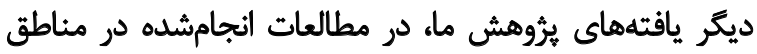

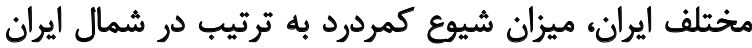

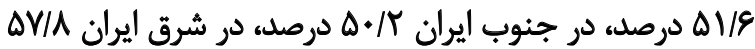

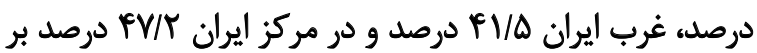

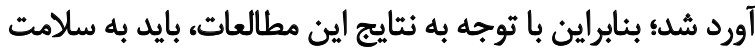

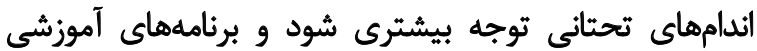

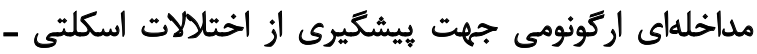

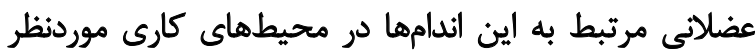
مديران و مسئولين سازمان ها قرار كيرد.

ديكر نتايج مطالعه ما نشان داد شيوع اختلالات اسكلتى ـ

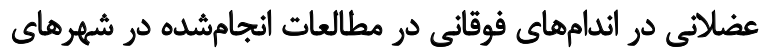

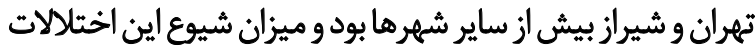

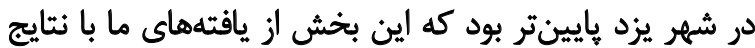

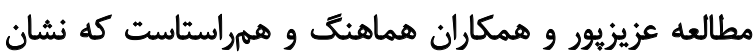

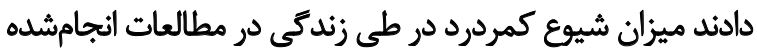
آنها در شهرهاى تهران و شيراز بالاتر از مطالعه انجامشدهد در شردير
اين ناحيه در بين كاركنان ايرانى است. ليوّ و همكاران نيز در

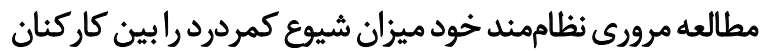

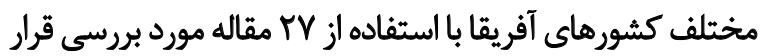

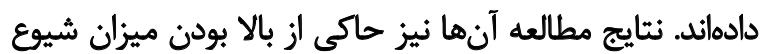

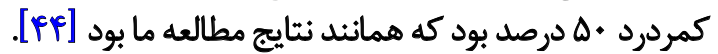
افزون بر اين، نتايج مطالعه هوو و و همكاران نيز نشان داد

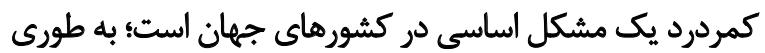

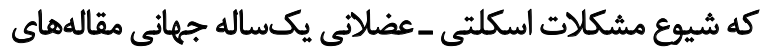

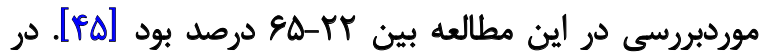

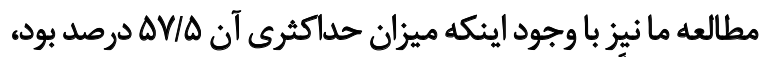

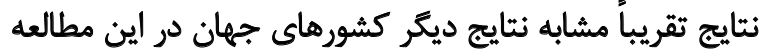

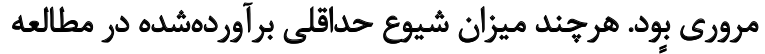

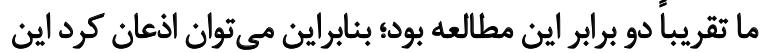

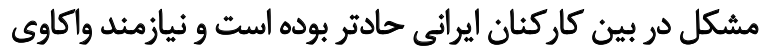
بيشتر براى بررسى علل اين شيوع است.

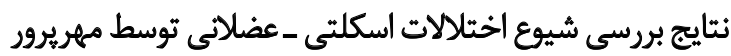

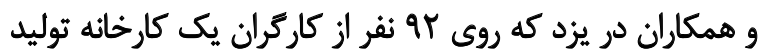

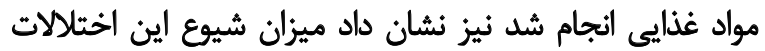

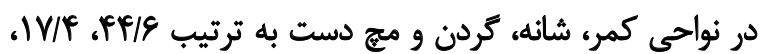

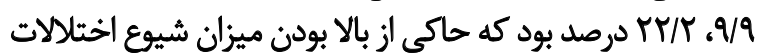

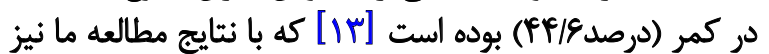
مطابقت داشت. از طرفى مطالعه اوسبورنه و همكاران نيز نشان آنان 


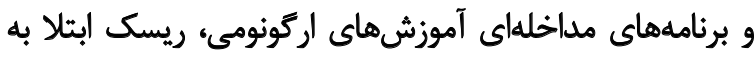

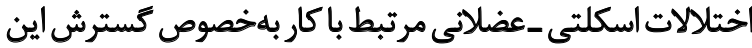

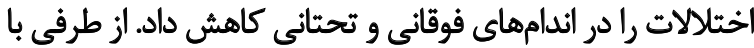

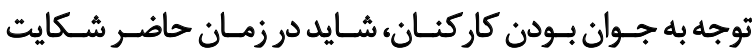

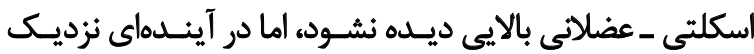

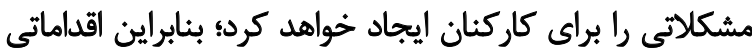

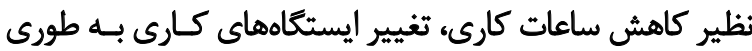

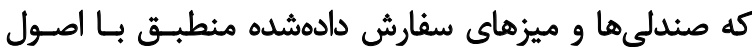

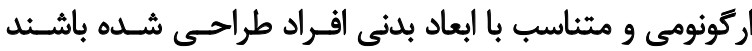

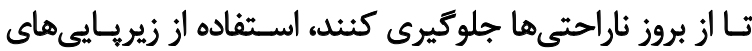

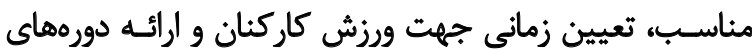

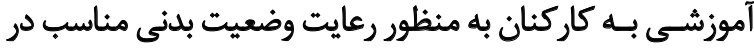

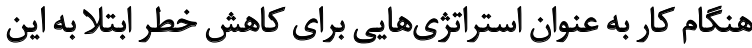

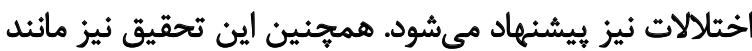

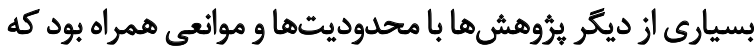

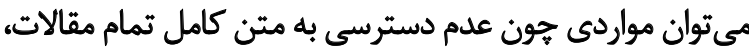

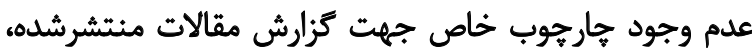

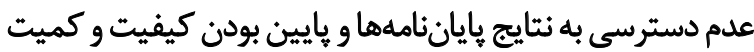
بانكهاى اطلاعاتي اشاره كرد.

ماحظات اخلاقي - ماتي

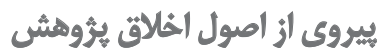

كليـهـ اصـول اخلاقـى مربـوط جــاب مقالـه مـرورى رعايـت

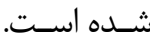

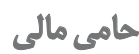

اين مقاله هيج نوع حامى مالى ندارد.

مشاركت ثويسند أنان

تمامى نويسند

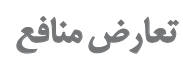

نويسندكان اظهار مى الرند هيجگَونه تعارض منافعى در مورد

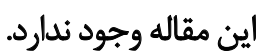

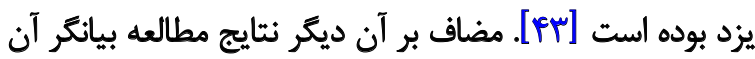

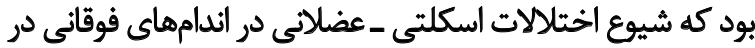

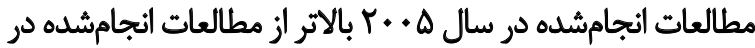

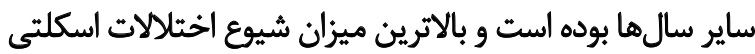

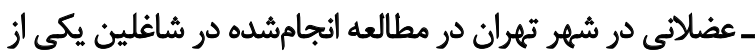

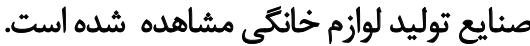

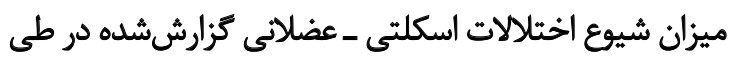

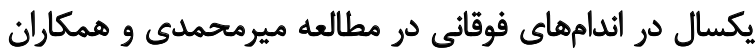

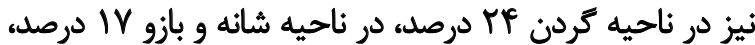

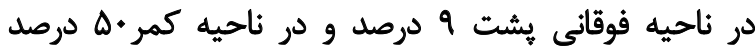

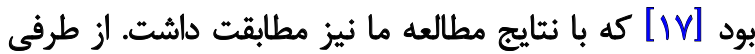

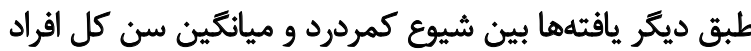

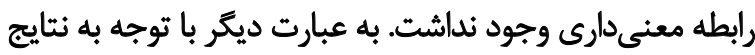

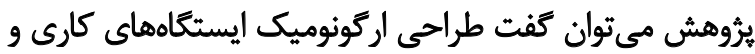

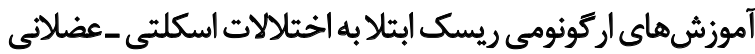

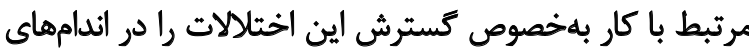

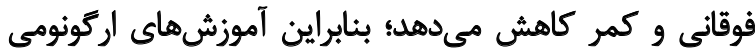

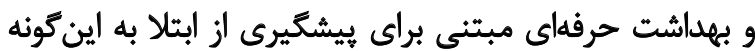

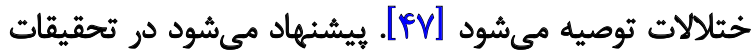

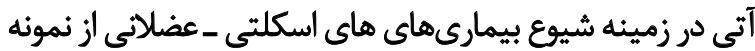

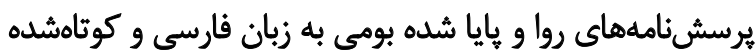

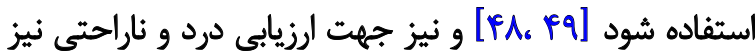

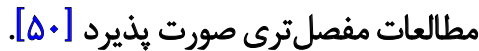
نتيجليَيرى

درمجموع نثتايج مطالعه حاكى از آن است كه مشكلات اسكلتى

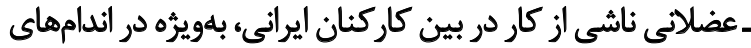

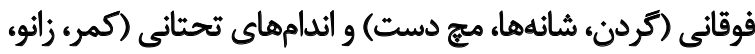

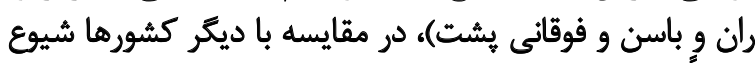

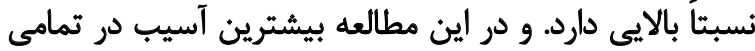

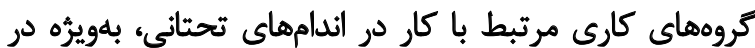

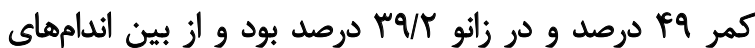

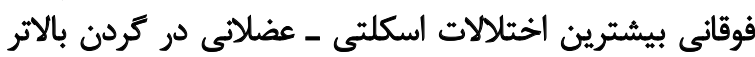

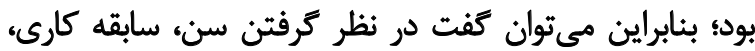

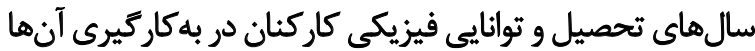

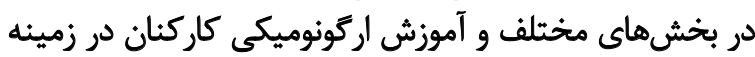

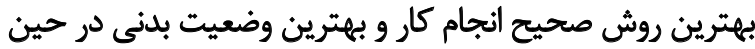

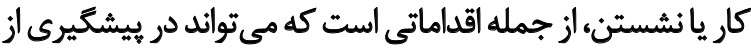
اختلالات اسكلتى ـ عضلانى نقش مؤثرى ايفا كند. بر اين اساس

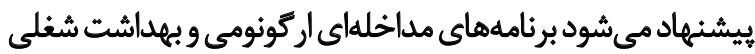

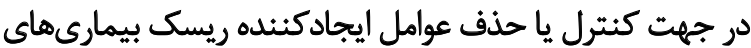
شغلى در مشاغل مختلف اجرا شود.

همجنين مى توان با طراحى اركونوميك ايستًاههاى كارى 


\section{References}

[1] National Institute for Occupational Safety and Health (NIOSH). Work-related musculoskeletal disorders (NIOSH Facts, document No. 705005). Washington DC.: NIOSH; 1997.

[2] Parno A, Sayehmiri K, Parno M, Khandan M, Poursadeghiyan M, Maghsoudipour M, et al. The prevalence of occupational musculoskeletal disorders in Iran: A meta-analysis study. Work. 2017; (58):203-214. [DOI:10.3233/WOR-172619] [PMID]

[3] Khandan M, Koohpaei A, Kohansal Aghchay M, Ebrahimi MH, Khammar A, Arsang Jang S. [Assessing the factors predicting work-related musculoskeletal disorders among Iranian port's personnel using regression model (Persian)]. Iranian Rehabilitation Journal. 2017; 15(4):309-16. [DOI:10.29252/nrip.irj.15.4.309]

[4] Khandan M, Vosoughi S, Poursadeghiyan M, Azizi F, Ahounbar E, Koohpaei A. [Ergonomic assessment of posture risk factors among Iranian Workers: An alternative to conventional methods (Persian)]. Iranian Rehabilitation Journal. 2018; 16(1):11-6. [DOI:10.29252/nrip.irj.16.1.11]

[5] Azadi F, Amjad RN, Marioryad H, Alimohammadi M, Karimpour Vazifehkhorani A, Poursadeghiyan M. [Effect of 12-week neck, core, and combined stabilization exercises on the pain and disability of elderly patients with chronic non-specific neck Pain: A clinical trial (Persian)]. Salmand: Iranian Journal of Ageing. 2019; 13(5):614-25. [DOI:10.32598/SIJA.13.Special-Issue.614]

[6] International Labor Organization (ILO). Recommendation concerning the list of occupational diseases and the recording and notification of occupational accidents and diseases (Recommendation R194). Geneva; ILO. 2002. https://www.ilo.org/dyn/normlex/en/f?p=NORMLEXPUB:12100:0::NO::P12100_INSTRUMENT_ID:312532

[7] Weahrer G, Leigh P, Miller T. Costs of occupational injury and illness within the health service sector. International Journal of Health Services. 2005; 35(2):243-359. [DOI:10.2190/RNQ30C13-U09M-TENP] [PMID]

[8] Cooper C, Marshall J. Sources of managerial and white collar stress. I CL Cooper \& R. Payne (Red.), Stress at Work. 1978. https://www.semanticscholar.org/paper/Sources-of-managerialand-white-collar-stress-Cooper-Marshall/db075d3702ad52c4b829f82da171f42bc770199b

[9] Croasmun J. Robotic legs may make difficult tasks easier. The trusted source for ergonomics. Ergonomics today [Internet]. 2004 [Updated 2004 Mar 24]. Available from: https://bleex.me.berkeley. edu/wp-content/uploads/hel-media/bleexhistPDFs/ergonomics. pdf

[10] Koohpaei A, Khandan M, Vosoughi S, Khammar A, Mobinizade V, Farrokhi M, et al. Industrial workers' postures analysis by a new method named "loading on the upper body assessment" in Iran. Annals of Tropical Medicine and Public Health. 2017; 10(4):97377. [DOI:10.4103/ATMPH.ATMPH_304_17]

[11] Moradi M, Poursadeghiyan M, Khammar A, Hami M, Darsnj A, Yarmohammadi H. REBA method for the ergonomic risk assessment of auto mechanics postural stress caused by working conditions in Kermanshah (Iran). Annals of Tropical Medicine and Public Health. 2017; 10(3):589. [DOI:10.4103/ATMPH.ATMPH_303_17]
[12] Choobineh A, Lahmi M, Shahnavaz H, Khani Jazani R, Hosseini M. Musculoskeletal symptoms as related to ergonomic factors in Iranian hand-woven carpet industry and general guidelines for workstation design. International Journal of Occupational Safety and Ergonomics. 2004; 10(2):157-68. [DOI:1 0.1080/10803548.2004.11076604] [PMID]

[13] Mehrparvar A, Ranjbar S, Mostaghaci M, Salehi M. Risk assessment of musculoskeletal disorders by QEC method in a food production factory. Occupational Medicine Quarterly Journal. 2011; 3(2):54-60. http://tkj.ssu.ac.ir/article-1-46-fa.html

[14] Steiner M. Postnatal depression: A few simple questions. Family Practice. 2002; 19(5):469-70. [DOI:10.1093/fam$\mathrm{pra} / 19.5 .469]$ [PMID]

[15] Halvani G, Salmani Nodousgan Z. [Survey of Musculoskeletal disorders among bank staff in Yazd (Persian)]. Occupational Medicine Quarterly Journal. 2011; 3(1):1-7. http://tkj.ssu ac.ir/article-1-48-fa.htm

[16] Choobineh A, Hosseini M, Lahmi M, Khani Jazani R, Shahnavaz H. Musculoskeletal problems in Iranian hand-woven carpet industry in Iran. Applied Ergonomics. 2007; 38(5):617-24. [DOI:10.1016/i. apergo.2006.06.005]

[17] Mirmohamadi M, Seraji JN, Shahtaheri J, Lahmi M, Ghasemkhani M. Evaluation of risk factors causing musculoskeletal disorders using QEC method in a furniture producing unite. Iranian Journal of Public Health. 2004; 33(2):24-7. https://ijph.tums.ac.ir/index.php/ ijph/article/view/1912

[18] Saremi M, Lahmi M, Faghihzadeh S. [The effect of ergonomic in tervention on dentists' musculoskeletal disorders (Persian)]. Daneshvar Medicine. 2006; 13(64):55-62. https://www.sid.ir/en/journal/ ViewPaper.aspx?id $=66296$

[19] Keshtkaran A, Daniali A. [Ergonomics disorders in the personnel of medical records department at training hospitals of Shiraz university of medical sciences (Persian)]. Health Information Management. 2007; 4(7):61-9. https://www.sid.ir/en/journal/ViewPaper. aspx?id $=112479$

[20] Ghasemkhani M, Rahimi M, Mosayeb Zadeh M. [Musculoskeletal symptom survey among cement drillers (Persian)]. Iran Occupational Health. 2005; 2(1):28-31. http://ioh.iums.ac.ir/article-1-78-fa.html

[21] Choubineh AR, Mokhtarzadeh A, Salehi M, Tabatabaei SHR. [Ergonomic evaluation of exposure to musculoskeletal disorders risk factors by qec technique in a rubber factory (Persian)]. Jundishapur Scientific Medical Journal. 2008; 7(56):46-55. https://www.sid.ir/en/ journal/ViewPaper.aspx?id $=123560$

[22] Choobineh A, Nouri E, Arjmandzadeh A, Mohamadbaigi A. [Musculoskeletal disorders among bank computer operators (Persian)] Iran Occupational Health. 2006; 3(2):3. http://ioh.iums.ac.ir/article1-472-fa.html

[23] Nasl Seraji J, Hajaghazadeh Firvaragh M, Hosseini SM, Adl J. [Musculoskeletal disorders study in a construction industry workers (Persian)]. Iran Occupational Health. 2007; 4(1-2):15-9. http://ioh.iums. ac.ir/article-1-458-en.pdf

[24] Choobineh A, Solaymani E, Mohammad Beigi A. [Musculoskeletal symptoms among workers of metal structure manufacturing industry in Shiraz, 2005 (Persian)]. Iranian Journal of Epidemiology. 2009; 5(3):35-43. https://irje.tums.ac.ir/article-1-108-en.pdf 
[25] Khosroabadi A, Razavi S, Fallahi M, Akaberi A. [The prevalence of musculoskeletal disorders in health-treatment employees at $\mathrm{Sa}$ bzevar University of Medical Sciences, Iran in 2008 (Persian)]. Journal of Sabzevar University of Medical Sciences. 2010; 17(3):218-23. http://jsums.medsab.ac.ir/article_59_ef0c50e7240285b4c97abb188f164ed7.pdf

[26] Rahimifard H, Hashemi Nejad N, Choobineh A, Haidari H, Tabatabaei $\mathrm{H}$. [Assessment of risk factors and prevalence of musculoskeletal disorders in raw furniture preparation workshops of the furniture industry (Persian)]. Journal of School of Public Health and Institute of Public Health Research. 2010; 8(1):53-68. https://sjsph.tums.ac.ir/ article-1-94-fa.pdf

[27] Barkhordari A, Jafari Nodoushan R, Vatani Shoaa J, Halvani G, Salmani Nodoushan M. [Posture evaluation using OWAS, RULA, QEC Method in FERO-ALEAGE factory workers of kerman (Persian)]. Occupational Medicine Quarterly Journal. 2011; 2(1):14-9. http://tkj. ssu.ac.ir/browse.php?mag_id=5\&slc_lang=en\&sid=1

[28] Haghdoost AA, Hajihosseini F, Hojjati H. [Relationship between the musculoskeletal disorders with the ergonomic factors in nurses (Persian)]. Koomesh. 2011; 12(4):372-8. http://koomeshjournal.semums. ac.ir/article-1-1347-fa.html

[29] Mirmohammadi S, Mehrparvar A, Soleimani H, Lotfi MH, Akbari H, Heidari N. [Musculoskeletal disorders among Video Display Terminal (VDT) workers comparing with other office workers (Persian)]. Iran Occupational Health. 2010; 7(2):11-4. http://ioh.iums.ac.ir/article1-285-fa.pdf

[30] Aminian O, Pouryaghoub G, Shanbeh M. One year study of musculoskeletal disorders and their relation to occupational stress among office workers: A brief report. Tehran University Medical Journal. 2012; 70(3):194-9. http://tumj.tums.ac.ir/article-1-138-en.html

[31] Mohammadfam I, Kianfar A, Afsartala B. [Assessment of musculoskeletal disorders in a manufacturing company using QEC and LUBAmethods and comparison of results (Persian)]. Iran Occupational Health. 2010; 7(1):7. http://ioh.iums.ac.ir/article-1-282-fa.pdf

[32] Habibi E, Gharib S, Shakerian M, Hasanzadeh A. [The prevalence of musculoskeletal disorders and analyzing the ergonomic status of workers involved manually carrying goods in the dairy industry (Persian)]. Journal of Health System Research. 2011; 6(4):649-57. https:// hsr.mui.ac.ir/article-1-187-fa.pdf

[33] Soltani R, Dehghani Y, Sadeghi Naeini H, Falahati M, Zokai M. [The welders posture assessment by owas technique (Persian)]. Occupational Medicine. 2011; 3(1):34-9. http://tkj.ssu.ac.ir/article-1-53-fa.html

[34] Eskandari D, Ghahri A, Gholamie A, Motalebi Kashani M, Mousavi SGA. [Prevalence of musculoskeletal disorders and work-related risk factors among the employees of an automobile factory in Tehran during 2009-10 (Persian)]. KAUMS Journal (FEYZ). 2011; 14(5):539-45. http:// feyz.kaums.ac.ir/article-1-1091-fa.pdf

[35] Tajvar A, Hasheminejad N, Bahrampour A, Chubineh A, Jalali A. Musculoskeletal disorders among small trades workers: A survey in the bakeries. Hormozgan Medical Journal. 2012; 15(4):e88504. https:// sites.kowsarpub.com/hmj/articles/88504.html

[36] Khani Jazani R, Fallah H, Barkhordari A, Halvani GH, Hokmabadi RA. The prediction of the incidence rate of upper limb musculoskeletal disorders, with CTD risk index method on potters of Meybod City. Zahedan Journal of Research in Medical Sciences. 2012; 13(9):e93733. https:// sites.kowsarpub.com/zjrms/articles/93733.html
[37] Choobineh A, Movahed M, Tabatabaie SH, Kumashiro M. Perceived demands and musculoskeletal disorders in operating room nurses of Shiraz city hospitals. Industrial Health. 2010; 48(1):74-84. [DOI:10.2486/indhealth.48.74] [PMID]

[38] Gharahgouzlou F, Karami Matin B. Ergonomic assessment of manual material handling and work - related musculoskeletal disorders in miners (kermanshah, 2001). Journal of Kermanshah University of Medical Sciences (Behbood). 2002; 6(1):e80964. https://sites.kowsarpub.com/jkums/articles/80964.html

[39] Nasl Seraji J, Zeraati H, Pouryaghoub GR, Gheybi L. [Musculoskeletal disorders study in damming construction workers by fox equation and measurement heart rate at work (Persian)]. Iran Occupational Health. 2008; 5(1-2):55-60. http://ioh.iums.ac.ir/ article-1-122-fa.pdf

[40] Ghamari F, Mohammadbeigi A, Khodayari M. [Work stations revision by ergonomic posture analyzing of Arak bakery workers (Persian)]. Journal of Advances in Medical and Biomedical Research. 2010; 18(70):80-90. http://zums.ac.ir/journal/article-1-1087-fa.pdf

[41] Rahimifard H, Nejad N, Choobineh A, Heidari H, Tabatabaei H. [Evaluation of musculoskeletal disorders risk factors in painting workshops of furniture industry (Persian)]. Qom University of Medical Sciences Journal. 2010; 4(2):45-54. http://journal.muq ac.ir/article-1-56-fa.html

[42] Parno A, Sayehmiri K, mokarami H, parno M, Azrah K, Ebrahimi $\mathrm{MH}$, et al. [The prevalence of work-related musculoskeletal disorders in the lower limbs among Iranian workers: A meta-analysis study (Persian)]. Iran Occupational Health. 2016; 13(5):50-9. http://ioh.iums.ac.ir/article-1-1435-fa.pdf

[43] Azizpoor Y, Hemmati F, Sayehmiri K. PPrevalence of life-time back pain in Iran: A systematic review and meta-analysis (Persian)]. Scientific Journal of Kurdistan University of Medical Sciences. 2013; 18(4):102-12. http://sjku.muk.ac.ir/article-1-1193-fa.pdf

[44] Louw QA, Morris LD, Grimmer-Somers K. The prevalence of low back pain in Africa: A systematic review. BMC Musculoskeletal Disorders. 2007; 8(1):105. [DOI:10.1186/1471-2474-8-105] [PMID] [PMCID]

[45] Hoe VC, Urquhart DM, Kelsall HL, Sim MR. Ergonomic design and training for preventing work-related musculoskeletal disorders of the upper limb and neck in adults. Cochrane Database of Systematic Reviews. 2012; 2012(8). [DOI:10.1002/14651858. CD008570.pub2] [PMID] [PMCID]

[46] Osborne A, Blake C, Fullen BM, Meredith D, Phelan J, McNamara J, et al. Prevalence of musculoskeletal disorders among farmers: A systematic review. American Journal of Industrial Medicine. 2012; 55(2):143-58. [DOI:10.1002/ajim.21033] [PMID]

[47] Yarmohammadi H, Niksima S H, Yarmohammadi S, Khamma A, Marioryad H, Poursadeqiyan M. Evaluating the prevalence of musculoskeletal disorders in drivers systematic review and metaanalysis. Journal of Health and Safety at Work. 2019; 9(3):221-30. http://ihsw.tums.ac.ir/article-1-6168-en.html

[48] Mohammadi HY, Sohrabi Y, Poursadeghiyan M, RostamiR, Rahmani Tabar A, Abdollahzadeh D, Rahmani Tabar F. Comparing the posture assessments based on RULA and QEC methods in a carpentry workshop. Research Journal of Medical Sciences. 2016; 10(3):80-3. http://docsdrive.com/pdfs/medwelljournals/ rjmsci/2016/80-83.pdf 
[49] Poursadeqiyan M, Arefi MF, Khaleghi S, Moghadam AS, Mazloumi E, Raei M, et al. Investigation of the relationship between the safety climate and occupational fatigue among the nurses of educational hospitals in Zabol. Journal of Education and Health Promotion. 2020; 9:238. https://www.jehp.net/article. asp?issn $=2277-9531$; year $=2020$; volume $=9 ;$ issue $=1$; page $=238$; epag $\mathrm{e}=238$; aulast $=$ Poursadeqiyan

[50] Omidianidost A, Hosseini S, Jabari M, Poursadeghiyan M, Dabirian M, Charganeh SS, et al. The relationship between individual, occupational factors and LBP (Low Back Pain) in one of the auto parts manufacturing workshops of Tehran in 2015. Journal of Engineering and Applied Sciences. 2016; 11(5):1074-7. [DOI:10.36478/jeasci.2016.1074.1077] 